\title{
INVARIANT DIFFERENTIAL OPERATORS
}

\author{
BY \\ W. SMOKE
}

Introduction. Let $B$ be a differentiable manifold and let $\mathscr{V}$ and $\mathscr{W}$ be the sheaves of germs of differentiable sections of a pair $\boldsymbol{V}$ and $\boldsymbol{W}$ of differentiable vector bundles on $B$. A problem that has had recent attention, notably from Spencer [5], is that of constructing a resolution of the kernel, or solution sheaf, of a differential operator $\mathscr{D}: \mathscr{V} \rightarrow \mathscr{W}$. We consider this problem in the case where $B$ is the homogeneous space $G / H$ of a Lie group $G$ modulo a closed subgroup $H$, with $\boldsymbol{V}$ and $\boldsymbol{W}$ homogeneous vector bundles, and under the assumption that $\mathscr{D}$ is invariant under the natural action of $G$ on the sheaves.

Invariant differential operators have been studied by Bott [1] in connection with the Atiyah-Singer index formula. In the special case where $\mathscr{V}=\mathscr{W}$ is a sheaf of germs of differentiable functions, they generalize the constant-coefficient differential operators of analysis, and have been studied by many authors. See, e.g., Helgason [3], where further references may be found.

In the case of an invariant differential operator, one would like a resolution of its solution sheaf which leads to Lie algebra cohomology. In keeping with this aim, and as a matter of general interest, we find it useful to transfer the basic formal apparatus of jet bundles, etc., to the Lie algebra setting. Thus, if $\boldsymbol{V}$ is a homogeneous vector bundle associated to an $H$-module $V$, one would expect the $q$ th jet bundle $V_{q}$ of $\boldsymbol{V}$ to be homogeneous. This is the case: there is an $\boldsymbol{H}$-module $J_{q}\left(\mathfrak{g}^{*}\right) \otimes^{\mathfrak{h}} V$, defined in terms of the Lie algebras $\mathfrak{g}$ and $\mathfrak{h}$, to which $V_{q}$ is associated. The $H$-modules $J_{q}\left(\mathfrak{g}^{*}\right) \otimes^{\mathfrak{h}} V$ have an inverse limit $J\left(\mathfrak{g}^{*}\right) \otimes^{\mathfrak{h}} V$, which is a $\mathfrak{g}$-module. Modifying a definition of Hochschild [4], we call an element of $J\left(\mathfrak{g}^{*}\right) \otimes^{\mathfrak{h}} V$ representative if it generates a finite-dimensional semisimple submodule of $J\left(\mathfrak{g}^{*}\right) \otimes \mathfrak{h} V$ under the action of $\mathfrak{g}$. The representative elements form a submodule $R\left(g^{*}\right) \otimes^{\mathfrak{h}} V$ which decomposes

$$
R\left(\mathfrak{g}^{*}\right) \otimes^{\mathfrak{h}} V \simeq \sum_{I} M_{i} \otimes \operatorname{Hom}_{\mathfrak{h}}\left(M_{i}, V\right)
$$

if the field is algebraically closed. Here, $I$ indexes a complete family of inequivalent finite-dimensional simple $\mathfrak{g}$-modules.

With $H$ connected, it turns out that the $G$-invariant differential operators $\mathscr{D}: \mathscr{V} \rightarrow \mathscr{W}$ are in 1-1 correspondence with certain $\mathfrak{g}$-invariant homomorphisms

$$
D: J\left(\mathrm{~g}^{*}\right) \otimes^{\mathfrak{h}} V \rightarrow J\left(\mathrm{~g}^{*}\right) \otimes^{\mathfrak{h}} W .
$$

Received by the editors June 7, 1965. 
The kernel $K_{R}$ of $D$ on $R\left(\mathrm{~g}^{*}\right) \otimes^{\mathfrak{h}} V$ decomposes $K_{R}=\sum_{I} K_{i}$. A similar decomposition $\mathscr{K}_{R}=\sum_{I} \mathscr{K}_{i}$ holds for the representative part of the kernel of $\mathscr{D}$, and $\mathscr{K}_{i}$ has a resolution by sheaves of germs of differential forms with values in the homogeneous vector bundle associated to $K_{i}$. The de Rham theorem applies, and, assuming $G$ compact and connected, the cohomology of $B$ with coefficients in $\mathscr{K}_{i}$ is $H\left(\mathfrak{g}^{*}, \mathfrak{h}^{*}\right) \otimes K_{i}$ or zero, according to whether or not the representation of $\mathfrak{g}$ on $M_{\mathfrak{i}}$ is induced by a representation of $G$. Here, $H\left(\mathfrak{g}^{*}, \mathfrak{h}^{*}\right)$ is the relative Lie algebra cohomology.

The procedure is therefore a generalization of the classical method for reducing the cohomology of $B$ with coefficients in the constant sheaf to $H\left(g^{*}, \mathfrak{h}^{*}\right)$, as given in Chevalley-Eilenberg [2]. To recover the classical result, take $\mathscr{D}$ to be the exterior differential, $\mathscr{V}$ the sheaf of germs of differentiable complex functions on $B$.

Chapter I is a review of the fundamentals of jet bundles and differential operators. Chapter II sets up the corresponding Lie-algebraic machinery, and Chapter III introduces homogeneous vector bundles and invariant differential operators, connecting the results of the first two chapters. The cohomological results appear in Chapter IV.

This paper is a revised form of my thesis, and I would like to express my gratitude to my advisers S. S. Chern and P. A. Griffiths. My studies were supported by the National Science Foundation under grant NSF GP-1610.

\section{Differential Operators}

1. Jet bundles. Let $F$ be the field of real or complex numbers. Let $B$ be a differentiable manifold, and let $\mathscr{F}$ be the sheaf of germs of differentiable $F$-valued functions on $B$. A tangent vector field on $B$ is a derivation of the algebra $\Gamma \mathscr{F}$ of sections of $\mathscr{F}$. The tangent bundle $\boldsymbol{T}$ of $B$ therefore depends on the field $F$, and is a complex vector bundle if $F$ is the complex field.

Let $\mathscr{F}(b)$ be the stalk of $\mathscr{F}$ at $b \in B$. The homomorphism from $\Gamma \mathscr{F}$ into $F$ defined by $f \rightarrow f(b)$ induces an augmentation $\varepsilon: \mathscr{F}(b) \rightarrow F$ with kernel $\mathscr{I}(b)$. The algebra $\mathscr{F}(b)$ is filtered by the powers $\mathscr{I}^{q}(b)$ of the ideal $\mathscr{I}(b)$, and the quotient algebra $\boldsymbol{F}_{q}(b)=\mathscr{F}(b) / \mathscr{I}^{q+1}(b)$ is called the algebra of $q$-jets at $b$. We write $j_{q, b}: \mathscr{F}(b) \rightarrow \boldsymbol{F}_{q}(b)$ for the natural homomorphism. The graded algebra associated to the filtered algebra $\mathscr{F}(b)$ is denoted by $\boldsymbol{S}(b)=\sum \boldsymbol{S}^{q}(b)$, so that the homogeneous component $\boldsymbol{S}^{q}(b)$ is the quotient space $\mathscr{I}^{a}(b) / \mathscr{I}^{q+1}(b)$. The inclusions between the ideals yield an exact sequence

$$
0 \longrightarrow \boldsymbol{S}^{q}(b) \stackrel{i_{q}}{\longrightarrow} \boldsymbol{F}_{q}(b) \stackrel{\pi_{q}}{\longrightarrow} \boldsymbol{F}_{q-1}(b) \longrightarrow 0
$$

for every $q$.

We introduce some useful notation. Let $B$ be of dimension $n$, let $g_{1}, \ldots, g_{n}$ be coordinate functions which define a chart at $b$, and let $Y_{1}, \ldots, Y_{n}$ be tangent vector fields which are locally dual to the coordinate functions, so that $Y_{i} g_{j}=\delta_{i j}$. For every sequence $r=\left(r_{1}, \ldots, r_{n}\right)$ of $n$ nonnegative integers, let $g^{r}$ be the monomial 
$g_{1}^{r_{1}} \cdots g_{n}^{r_{n}}$ and let $Y^{r}$ be the product $Y_{1}^{r_{1}} \cdots Y_{n}^{r_{n}}$. Let also $r !=r_{1} ! \cdots r_{n}$ ! and $|r|$ $=r_{1}+\cdots+r_{n}$. We may suppose that $g_{1}, \ldots, g_{n}$ vanish at $b$, so that their germs $\gamma_{1}, \ldots, \gamma_{n}$ belong to $\mathscr{I}(b)$. A tangent vector field defines a derivation of $\mathscr{F}(b)$, and it is not difficult to see that $Y^{r} \gamma^{r}=r$ !, while $Y^{r} \gamma^{s}$ is an element of $\mathscr{I}(b)$ for $r \neq s$.

LEMMA 1.1. An element $\phi \in \mathscr{F}(b)$ belongs to $\mathscr{I}^{a}(b)$ if and only if $X_{1} \cdots X_{p} \phi \in \mathscr{I}(b)$ for every sequence of vector fields $X_{1}, \ldots, X_{p}$ such that $p<q$.

Proof. Since a vector field is a derivation of $\mathscr{F}(b)$, it maps $\mathscr{I}^{q}(b)$ into $\mathscr{I}^{q-1}(b)$. Thus $X_{1} \cdots X_{p}$ maps $\mathscr{I}^{q}(b)$ into $\mathscr{I}^{q-p}(b)$, which is contained in $\mathscr{I}(b)$ if $p<q$. To prove the converse inclusion, it will evidently be sufficient to show that an element $\phi \in \mathscr{I}^{p}(b)$ belongs to $\mathscr{I}^{p+1}(b)$ whenever $Y^{r} \phi \in \mathscr{I}(b)$ for $|r| \leqq p$. By the mean value theorem, an element $\varphi \in \mathscr{I}(b)$ may be written $\varphi=\varphi_{1} \gamma_{1}+\cdots+\varphi_{n} \gamma_{n}$ for some elements $\varphi_{1}, \ldots, \varphi_{n} \in \mathscr{F}(b)$. Thus $\phi \in \mathscr{I}^{p}(b)$ may be written

$$
\phi=\sum_{|s|=p} \phi_{s} \gamma^{s}
$$

for some $\phi_{s} \in \mathscr{F}(b)$. By assumption, $Y^{r} \phi \in \mathscr{I}(b)$ if $|r|=p$. Also, $Y^{r}\left(\phi_{s} \gamma^{s}\right) \in \mathscr{I}(b)$ if $r \neq s$, as is easily seen by expanding by Leibnitz's rule. Finally, every term in the expansion of $Y^{r}\left(\phi_{r} \gamma^{r}\right)$, with the possible exception of $\phi_{r} Y^{r} \gamma^{r}=\phi_{r} r$ !, also belongs to $\mathscr{I}(b)$, and it follows that $\phi_{r}$ must belong to $\mathscr{I}(b)$. Since this is true of each of the coefficients $\phi_{r}$ in (2), $\phi$ must belong to $\mathscr{I}(b) \mathscr{I}^{p}(b)=\mathscr{I}^{p+1}(b)$, and the lemma follows.

We have supposed that $g_{1}, \ldots, g_{n}$ are zero at $b$, so that their germs $\gamma_{1}, \ldots, \gamma_{n}$ belong to $\mathscr{I}(b)$. The following proposition holds without this assumption.

Proposition 1.2. If $\gamma_{1}, \ldots, \gamma_{n}$ are germs of coordinate functions which define a chart at $b \in B$, then $F_{q}(b)$ is a finite-dimensional vector space over $F$ with the basis $j_{q, b}\left(\gamma^{r}\right),|r| \leqq q$.

Proof. Suppose that $\sum_{|r| \leqq q} a_{r} j_{q, b}\left(\gamma^{r}\right)=0$. Then $\sum_{|r| \leqq q} a_{r} \gamma^{r}$ belongs to $\mathscr{I}^{q+1}(b)$. If $a_{r}$ is not zero for some $r$, choose $r$ with $a_{r} \neq 0$ and $|r|$ a maximum, and apply $Y^{r}$. This gives $a_{r} r ! \in \mathscr{I}(b)$, a contradiction, and the elements $j_{q, b}\left(\gamma^{r}\right)$ are independent. To see that they span $\boldsymbol{F}_{q}(b)$, suppose first that $\gamma_{1}, \ldots, \gamma_{n} \in \mathscr{I}(b)$. For any $\phi \in \mathscr{F}(b)$, the difference

$$
\phi-\sum_{|r| \leqq q} \frac{1}{r !}\left(Y^{r} \phi\right)(b) \cdot \gamma^{r}
$$

is mapped into $\mathscr{I}(b)$ by $Y^{r}$, for $|r| \leqq q$. Locally, every tangent vector field $X$ may be written $X=f_{1} Y_{1}+\cdots+f_{n} Y_{n}$, where the coefficients $f_{1}, \ldots, f_{n}$ are differentiable functions, and it follows that (3) is mapped into $\mathscr{I}(b)$ by any product $X_{1} \cdots X_{p}$ with $p \leqq q$. By the lemma, (3) belongs to $\mathscr{I}^{q+1}(b)$, so

$$
j_{q, b}(\phi)=\sum_{|r| \leqq q} \frac{1}{r !}\left(Y^{r} \phi\right)(b) j_{q, b}\left(\gamma^{r}\right)
$$

and the elements $j_{q, b}\left(\gamma^{r}\right)$ span $\boldsymbol{F}_{q}(b)$. Since they are in any case independent, they must therefore span $\boldsymbol{F}_{q}(b)$ whether the germs $\gamma_{1}, \ldots, \gamma_{n}$ belong to $\mathscr{I}(b)$ or not, and the proposition follows. 
Proposition 1.3. The graded algebra $\mathbf{S}(b)$ is naturally isomorphic to the symmetric algebra of the cotangent space $T(b)^{*}$.

Proof. Since $\phi \in \mathscr{F}(b)$ and $\phi-\phi(b) \in \mathscr{I}(b)$ have the same differential, the map $\phi \rightarrow(d \phi)(b)$ maps $\mathscr{I}(b)$ onto $T(b)^{*}$. An element $\phi \in \mathscr{I}(b)$ belongs to the kernel of this map if and only if $(X \phi)(b)=0$ for every tangent vector field $X$, thus if and only if $\phi \in \mathscr{I}^{2}(b)$. Thus $\boldsymbol{T}(b)^{*}$ is isomorphic to $S^{1}(b)$. Let $S\left(T(b)^{*}\right)$ be the symmetric algebra of $\boldsymbol{T}(b)^{*}$. Since $\boldsymbol{S}(b)$ is commutative, the vector space isomorphism extends to a homomorphism $S\left(\boldsymbol{T}(b)^{*}\right) \rightarrow \boldsymbol{S}(b)$, taking $\left(d \phi_{1}\right)(b) \cdots\left(d \phi_{q}\right)(b)$ into the class of $\left(\phi_{1}-\phi_{1}(b)\right) \cdots\left(\phi_{q}-\phi_{q}(b)\right)$ modulo $\mathscr{I}^{q+1}(b)$. Returning to the notation of Proposition 1.2, suppose again that $\gamma_{1}, \ldots, \gamma_{n} \in \mathscr{I}(b)$. The composition of $S^{q}\left(T(b)^{*}\right)$ $\rightarrow S^{q}(b)$ and $i_{q}: \boldsymbol{S}^{q}(b) \rightarrow \boldsymbol{F}_{q}(b)$ then takes $\left(d \gamma_{1}\right)^{r_{1}} \cdots\left(d \gamma_{n}\right)^{r_{n}}$ into $j_{q, b}\left(\gamma^{r}\right)$ for every $r$ with $|r|=q$. But $\pi_{q}: \boldsymbol{F}_{q}(b) \rightarrow \boldsymbol{F}_{q-1}(b)$ maps $j_{q, b}\left(\gamma^{r}\right)$ into $j_{q-1, b}\left(\gamma^{r}\right)$, and it follows that the elements $j_{q, b}\left(\gamma^{r}\right),|r|=q$, form a basis of the kernel of $\pi_{q}$. Since (1) is exact, the proposition follows.

The inverse limit $\boldsymbol{F}_{\infty}(b)=\lim \operatorname{inv} \boldsymbol{F}_{q}(b)$ is called the jet algebra at $b$. The inverse limit of the homomorphisms $j_{q, b}$ is a homomorphism $j_{b}: \mathscr{F}(b) \rightarrow \boldsymbol{F}_{\infty}(b)$, and $j_{b}(\phi)$ is the jet of $\phi$. The homomorphisms $j_{q, b}$ and $j_{b}$ are extended to differentiable functions in the obvious way, and $f$ has a $q$-jet $j_{q, b}(f)$ and a jet $j_{b}(f)$ at $b$ whenever $f$ is differentiable in a neighborhood of $b \in B$.

Suppose now that $V$ is a differentiable vector bundle on $B$, real or complex according to whether $F$ is the real or complex field. Let $\mathscr{V}$ be the sheaf of germs of differentiable sections of $\boldsymbol{V}$. Then $\mathscr{V}$ is a sheaf of $\mathscr{F}$-modules, and the submodules $\mathscr{I}^{a}(b) \mathscr{V}(b)$ define a filtration on the stalk $\mathscr{V}(b)$, for $b \in B$. The quotient $\boldsymbol{V}_{q}(b)$ $=\mathscr{V}(b) / \mathscr{I}^{q+1}(b) \mathscr{V}(b)$ is the space of $q$-jets at $b$ with values in $\boldsymbol{V}$. The natural map $\mathscr{V}(b) \rightarrow \boldsymbol{V}_{q}(b)$ is again written $j_{q, b}$. The space $\boldsymbol{V}_{q}(b)$ is a module over $\boldsymbol{F}_{q}(b)$, with $j_{q, b}(\phi) j_{q, b}(\sigma)=j_{q, b}(\phi \sigma)$ for $\phi \in \mathscr{F}(b)$ and $\sigma \in \mathscr{V}(b)$. The sheaf $\mathscr{V}$ is locally free, and $\mathscr{V}(b)$ has a basis $\tau_{1}, \ldots, \tau_{m}$ over $\mathscr{F}(b)$. Thus $\sigma \in \mathscr{V}(b)$ may be uniquely written

$$
\sigma=\phi_{1} \tau_{1}+\cdots+\phi_{m} \tau_{m}
$$

with $\phi_{1}, \ldots, \phi_{m} \in \mathscr{F}(b)$, and

$$
j_{q, b}(\sigma)=j_{q, b}\left(\phi_{1}\right) j_{q, b}\left(\tau_{1}\right)+\cdots+j_{q, b}\left(\phi_{m}\right) j_{q, b}\left(\tau_{m}\right) .
$$

It follows that the elements $j_{q, b}\left(\tau_{i}\right)$ span $V_{q}(b)$ over $\boldsymbol{F}_{q}(b)$. If $j_{q, b}(\sigma)=0$ then $\sigma \in \mathscr{I}^{q+1}(b) \mathscr{V}(b)$, and it follows that the coefficients $\phi_{i}$ in (5) belong to $\mathscr{I}^{q+1}(b)$. Thus $j_{q, b}\left(\phi_{i}\right)=0$ for each $i$, hence the elements $j_{q, b}\left(\tau_{i}\right)$ form a basis of $V_{q}(b)$ over $\boldsymbol{F}_{q}(b)$. It follows from Proposition 1.2 that $\boldsymbol{V}_{q}(b)$ is a finite-dimensional vector space over $F$, with a basis consisting of the elements $j_{q, b}\left(\gamma^{r} \tau_{i}\right),|r| \leqq q, i=1, \ldots, m$.

Proposition 1.4. The graded space associated to $\mathscr{V}(b)$ is naturally isomorphic to $S(b) \otimes V(b)$, and there are exact sequences

$$
0 \longrightarrow S^{q}(b) \otimes V(b) \stackrel{i_{q}}{\longrightarrow} V_{q}(b) \stackrel{\pi_{q}}{\longrightarrow} V_{q-1}(b) \longrightarrow 0 .
$$


Proof. Define a map $i_{q}: \boldsymbol{S}^{q}(b) \otimes \boldsymbol{V}(b) \rightarrow \boldsymbol{V}_{q}(b)$ by associating to $\left(d \phi_{1}\right)(b) \cdots$ $\left(d \phi_{q}\right)(b) \otimes v$ the element $j_{q, b}\left(\phi_{1} \cdots \phi_{q} \sigma\right)$, choosing $\phi_{1}, \ldots, \phi_{q} \in \mathscr{I}(b)$ and $\sigma$ so that $\sigma(b)=v$. The value is independent of the choice of $\sigma$, for if also $\sigma^{\prime}(b)=v$ then $\sigma-\sigma^{\prime}$ is zero at $b$, and the products $\phi_{1} \cdots \phi_{q} \sigma$ and $\phi_{1} \cdots \phi_{q} \sigma^{\prime}$ are congruent modulo $\mathscr{I}^{a+1}(b) \mathscr{V}(b)$. Similarly, the value is independent of the choice of $\phi_{1}, \ldots, \phi_{a}$. If $\tau_{1}, \ldots, \tau_{m}$ is a basis of $\mathscr{V}(b)$ over $\mathscr{F}(b)$, then $v_{1}=\tau_{1}(b), \ldots, v_{m}=\tau_{m}(b)$ is a basis of $\boldsymbol{V}(b)$ over $F$, and $i_{q}$ takes the basis $\left(d \gamma_{1}\right)^{r_{1}} \ldots\left(d \gamma_{n}\right)^{r_{n}} \otimes v_{i}$ of the tensor product into a basis $j_{q, b}\left(\gamma^{r} \tau_{i}\right)$ of the kernel of $\pi_{q}$. The map $\pi_{q}$ is defined by $j_{q, b}(\sigma) \rightarrow j_{q-1, b}(\sigma)$ and is clearly onto, so the proposition is proved.

The space $\boldsymbol{V}_{\infty}(b)=\lim$ inv $\boldsymbol{V}_{q}(b)$ and the map $j_{b}: \mathscr{V}(b) \rightarrow \boldsymbol{V}_{\infty}(b)$ are defined as before, and extended to differentiable sections $s$ of $\boldsymbol{V}$. Let now $V_{q}=\bigcup_{B} V_{q}(b)$. Then $\boldsymbol{V}_{q}$ is a differentiable vector bundle on $B$, called the bundle of $q$-jets of $\boldsymbol{V}$. A differentiable section $s$ of $V$, defined on an open set $U$ in $B$, defines a section $j_{q}(s)$ of $V_{q}$ over $U$ by $j_{q}(s)(b)=j_{q, b}(s)$ for $b \in U$. Suppose that $U$ is a coordinate neighborhood over which $\boldsymbol{V}$ is trivial, with coordinate functions $g_{1}, \ldots, g_{n}$. Let $t_{1}, \ldots, t_{m}$ be a basis of differentiable sections of $V$ over $U$. It then follows from our earlier remarks that the sections $j_{q}\left(g^{r} t_{i}\right)$ are a basis of sections of $V_{q}$ over $U$, and the bundle $V_{q}$ has a unique differentiable structure such that the sections of the form $j_{q}\left(g^{t} t_{i}\right)$ are differentiable. To prove this, it must be verified that for every differentiable section $s$ of $V$ over $U$, the coefficients $f_{r i}$ in the expression

$$
j_{q}(s)=\sum_{i,|r| \geqq q} f_{r i} j_{q}\left(g^{r} t_{i}\right)
$$

are differentiable functions on $U$. Expressing $s$ in terms of the basis $t_{1}, \ldots, t_{m}$, $s=h_{1} t_{1}+\cdots+h_{m} t_{m}$, and expanding $h_{1}, \ldots, h_{m}$ according to (4), it results that the coefficients $f_{r i}$ are polynomials in the coordinate functions $g_{j}$ and the derivatives of the functions $h_{i}$. Thus the coefficients $f_{r i}$ are differentiable. The differentiable structure of $\boldsymbol{V}_{q}$ is therefore uniquely determined by the requirement that, for every differentiable section $s$ of $V$, the section $j_{q}(s)$ of $V_{q}$ be differentiable.

Proposition 1.5. For every $q$, there is an exact sequence

$$
\mathbf{0} \longrightarrow \boldsymbol{S}^{q} \otimes \boldsymbol{V} \stackrel{i_{q}}{\longrightarrow} \boldsymbol{V}_{q} \stackrel{\pi_{q}}{\longrightarrow} \boldsymbol{V}_{q-1} \longrightarrow \mathbf{0}
$$

of differentiable vector bundles and differentiable bundle homomorphisms.

Proof. The bundle $S^{q}$ is the bundle of symmetric differential forms of degree $q$ on $B$. From Proposition 1.4 it is clear that (7) is exact. The map $\pi_{q}: V_{q} \rightarrow V_{q-1}$ takes a section of the form $j_{q}(s), s$ a differentiable section of $V$, into $j_{q-1}(s)$, so is differentiable. The injection $i_{q}$ is given on a section of $\boldsymbol{S}^{q} \otimes \boldsymbol{V}$ by

$$
i_{q}\left(d f_{1} \cdots d f_{q} \otimes s\right)=\sum_{\sigma}(-1)^{p} f_{\sigma 1} \cdots f_{\sigma p} j_{q}\left(f_{\sigma(p+1)} \cdots f_{\sigma q} s\right),
$$

summed over all permutations $\sigma$ of $1, \ldots, q$ with the property that $\sigma(1)<\cdots<\sigma(p)$ and $\sigma(p+1)<\cdots<\sigma(q)$ for some $p, 1 \leqq p \leqq q$. For we have, evaluating at $b$, 
$i_{q}\left(d f_{1} \cdots d f_{q} \otimes s\right)(b)=j_{q, b}\left(\left(f_{1}-f_{1}(b)\right) \cdots\left(f_{q}-f_{q}(b)\right) s\right)$, which is the value at $b$ of the right-hand side of (8). Since the right-hand side of (8) is a differentiable section of $V_{q}, i_{q}$ is differentiable, and the proof is complete.

This proposition applies in particular to the trivial bundle $\boldsymbol{F}$ on $B$ with fiber $F$. The bundle $\boldsymbol{F}_{q}$ is the q-jet bundle of $B$.

2. Differential operators. Let $\boldsymbol{V}$ and $\boldsymbol{W}$ be differentiable vector bundles on $B$, and let $\mathscr{V}$ and $\mathscr{W}$ be the corresponding sheaves. Recall that a sheaf homomorphism $\mathscr{D}: \mathscr{V} \rightarrow \mathscr{W}$, linear over $F$, is a map which induces a vector space homomorphism $\mathscr{D}(b): \mathscr{V}(b) \rightarrow \mathscr{W}(b)$ for each $b \in B$, and takes local sections $s$ of $\mathscr{V}$ into local sections $\mathscr{D} s$ of $\mathscr{W}$. If $\mathscr{D}(b)$ maps $\mathscr{I}^{q}(b) \mathscr{V}(b)$ into $\mathscr{I}^{q-r}(b) \mathscr{W}(b)$ for each $q$ and each $b \in B$, then $\mathscr{D}$ is called a differential operator, of order $r$. It is immediate that the differential operators $\mathscr{D}: \mathscr{V} \rightarrow \mathscr{W}$ form a vector space over $F$. A differential operator of order $r$ is also of order $s$ for any $s$ greater than $r$, and the space of differential operators $\mathscr{D}: \mathscr{V} \rightarrow \mathscr{W}$ is filtered by order. The composition of two differential operators, when defined, is again a differential operator, of order the sum of the orders of the factors. Thus the differential operators $\mathscr{D}: \mathscr{V} \rightarrow \mathscr{V}$ form an algebra over $F$, filtered by order.

Suppose $\mathscr{D}: \mathscr{V} \rightarrow \mathscr{W}$ is a differential operator of order $r$. It is then immediate that the vector space homomorphism $\mathscr{D}(b): \mathscr{V}(b) \rightarrow \mathscr{W}(b)$ induces a vector space homomorphism $\boldsymbol{D}_{q}(b): \boldsymbol{V}_{q}(b) \rightarrow \boldsymbol{W}_{q-r}(b)$ for every $q$, given by $\boldsymbol{D}_{q}(b) j_{q, b}(\sigma)$ $=j_{q-r, b}(\mathscr{D} \sigma)$ for $\sigma \in \mathscr{V}(b)$. This is true for every $b \in B$, so to $\mathscr{D}$ is associated a bundle homomorphism $\boldsymbol{D}_{q}: \boldsymbol{V}_{q} \rightarrow \boldsymbol{W}_{q-r}$ for each $q$. If $s$ is a differentiable section of $V$, then $D_{q}$ is given on the differentiable section $j_{q}(s)$ of $V_{q}$ by

$$
\boldsymbol{D}_{q} j_{q}(s)=j_{q-r}(\mathscr{D} s)
$$

and it follows that the bundle homomorphism $\boldsymbol{D}_{q}$ is differentiable. For $q \geqq p$ the projection $V_{q} \rightarrow V_{p}$ takes $j_{q}(s)$ into $j_{p}(s)$, and the diagram

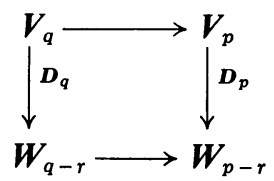

is commutative. Taking $p=q-1$, it follows that there is a bundle homomorphism $D^{q}$ such that

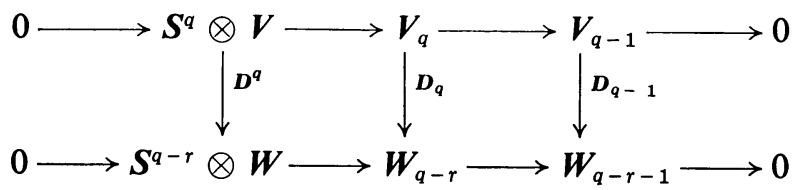

is commutative, called the $q$ th symbol of $\mathscr{D}$.

Since $V_{0}(b)=\mathscr{V}(b) / \mathscr{I}(b) \mathscr{V}(b)$, and since $\mathscr{I}(b) \mathscr{V}(b)$ contains the elements of $\mathscr{V}(b)$ which are zero at $b$, it follows that $V_{0}$ may be identified with $V$. The map $j_{0}$ 
is then the identity on sections of $\mathscr{V}$. Thus if $\mathscr{D}: \mathscr{V} \rightarrow \mathscr{W}$ is of order $r$, we have $D_{r} j_{r}(s)=\mathscr{D} s$ for any differentiable section $s$ of $\mathscr{V}$, by (1). Let $\mathscr{V}$, be the sheaf of germs of differentiable sections of the bundle $V_{r}$. It follows that $\mathscr{D}$ may be factored

$$
\mathscr{V} \stackrel{j_{r}}{\longrightarrow} \mathscr{V}_{r} \stackrel{\boldsymbol{D}_{\boldsymbol{r}}}{\longrightarrow} \mathscr{W} .
$$

Proposition 2.1. The map $j_{r}: \mathscr{V} \rightarrow \mathscr{V}_{r}$ is a differential operator of order $r$.

Proof. Clearly $j_{r}$ is a sheaf homomorphism, linear over $F$. We must show that $j_{r}$ maps $\mathscr{I}^{q}(b) \mathscr{V}(b)$ into $\mathscr{I}^{q-r}(b) \mathscr{V}_{r}(b)$ for every $q$. Since $j_{r}(\phi \sigma)=j_{r}(\phi) j_{r}(\sigma)$ for $\phi \in \mathscr{F}(b)$ and $\sigma \in \mathscr{V}(b)$, it will be sufficient to show that $j_{r}: \mathscr{F}(b) \rightarrow \mathscr{F}_{r}(b)$ maps $\mathscr{I}^{q}(b)$ into $\mathscr{I}^{q-r}(b) \mathscr{F}_{r}(b)$ for every $q$. Returning to the notation of Proposition 1.2, we have

$$
j_{r, b}(\phi)=\sum_{|s| \leqq r} \frac{1}{s !}\left(Y^{s} \phi\right)(b) j_{r, b}\left(\gamma^{s}\right)
$$

and it follows that the coefficients which appear in the expansion of $j_{r}(\phi)$ as a linear combination of the elements $j_{r}\left(\gamma^{s}\right)$ each contain a factor of the form $\left(Y^{s} \phi\right)$, where $|s| \leqq r$. But if $\phi \in \mathscr{I}^{q}(b)$ then $\left(Y^{s} \phi\right) \in \mathscr{I}^{q-r}(b)$ if $|s| \leqq r$, and it follows that $j_{r}(\phi) \in \mathscr{I}^{q-r}(b) \mathscr{F}_{r}(b)$, proving the proposition.

A differentiable vector bundle homomorphism, acting on the corresponding sheaves, is clearly a differential operator of order zero. Thus (2) is a factorization of $\mathscr{D}$ into the canonical differential operator $j_{r}$ of order $r$, and a differential operator of order zero. On the other hand, if $\boldsymbol{D}: \boldsymbol{V}_{r} \rightarrow \boldsymbol{W}$ is any differentiable bundle homomorphism then $\mathscr{D}=\boldsymbol{D} \cdot j_{r}$ is a differential operator $\mathscr{D}: \mathscr{V} \rightarrow \mathscr{W}$ of order $r$, and $\boldsymbol{D}_{r}=\boldsymbol{D}$. The relation $\boldsymbol{D}_{r} j_{r}=\mathscr{D}$ shows that the correspondence $\mathscr{D} \rightarrow \boldsymbol{D}_{r}$ is 1-1. This proves:

THEOREM 2.2. The correspondence $\mathscr{D} \rightarrow D_{r}$ is an isomorphism from the vector space of differential operators $\mathscr{D}: \mathscr{V} \rightarrow \mathscr{W}$ of order $r$ to the vector space of differentiable bundle homomorphisms $\mathrm{D}: V_{r} \rightarrow W$.

\section{The Jet Algebra of A Lie Algebra}

3. The jet algebra and its subalgebras. In this section the field $F$ may be any field of characteristic zero. Let $\mathfrak{g}$ be a finite-dimensional Lie algebra over $F$, and let $U(\mathrm{~g})$ be its universal enveloping algebra. Recall that $U(\mathrm{~g})$ is a coalgebra, in fact a Hopf algebra, with a diagonal map

$$
\Delta: U(\mathrm{~g}) \rightarrow U(\mathrm{~g}) \otimes U(\mathrm{~g})
$$

defined by extending $\Delta(x)=x \otimes 1+1 \otimes x, x \in \mathfrak{g}$, to an algebra homomorphism. For $x_{1}, \ldots, x_{q} \in \mathfrak{g}$ we have

$$
\Delta\left(x_{1} \cdots x_{q}\right)=\sum_{\sigma} x_{\sigma 1} \cdots x_{\sigma p} \otimes x_{\sigma(p+1)} \cdots x_{\sigma q}
$$

where the sum is over all shuffles of $1, \ldots, q$, i.e., all permutations $\sigma$ with the property that $\sigma(1)<\cdots<\sigma(p)$ and $\sigma(p+1)<\cdots<\sigma(q)$ for some $p, 1 \leqq p \leqq q$. The 
algebra $U(\mathfrak{g})$ is filtered by subspaces $U_{q}(\mathrm{~g})$, where $U_{q}(\mathrm{~g})$ is spanned by all products of at most $q$ elements of $\mathfrak{g}$. It follows from (1) that $\Delta$ maps $U_{q}(\mathfrak{g})$ into $U_{q}(\mathfrak{g}) \otimes U_{q}(\mathfrak{g})$, so that $U_{q}(\mathrm{~g})$ is a subcoalgebra of $U(\mathrm{~g})$.

By the Poincaré-Birkhoff-Witt Theorem, the graded algebra associated to $U(\mathrm{~g})$ is the symmetric algebra $S(\mathfrak{g})$ of $\mathfrak{g}$, and there are exact sequences

$$
0 \rightarrow U_{q-1}(\mathfrak{g}) \rightarrow U_{q}(\mathfrak{g}) \rightarrow S^{q}(\mathfrak{g}) \rightarrow 0
$$

Moreover, there exists a linear map $\lambda: S(\mathfrak{g}) \rightarrow U(\mathfrak{g})$ given by

$$
\lambda\left(x_{1} \cdots x_{q}\right)=\frac{1}{q !} \sum_{\sigma} x_{\sigma 1} \cdots x_{\sigma q}
$$

for $x_{1}, \ldots, x_{q} \in \mathfrak{g}$. The sum in (3) runs over all permutations. It is not difficult to see that $\lambda: S^{q}(\mathfrak{g}) \rightarrow U_{q}(\mathfrak{g})$ splits the sequence (2). If $S_{q}(\mathfrak{g})=\sum_{p \leqq q} S^{p}(\mathfrak{g})$ then $\lambda_{q}: S_{q}(\mathfrak{g}) \rightarrow U_{q}(\mathrm{~g})$ is therefore a vector space isomorphism. Thus $\lambda: S(\mathfrak{g}) \rightarrow U(\mathfrak{g})$ is a vector space isomorphism. The symmetric algebra $S(\mathfrak{g})$, like $U(\mathfrak{g})$, is a Hopf algebra, with diagonal map again satisfying (1), and the subspaces $S_{q}(\mathrm{~g})$ are subcoalgebras of $S(g)$. By counting permutations, one may conclude that

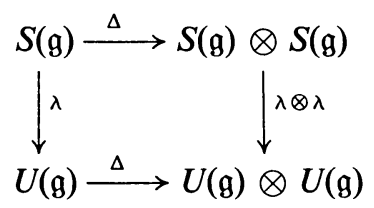

is commutative, so that $\lambda$ is a coalgebra isomorphism. The same is therefore true of $\lambda_{q}: S_{q}(\mathrm{~g}) \rightarrow U_{q}(\mathrm{~g})$.

The algebra $U(\mathrm{~g})$ has an involutive antiautomorphism $u \rightarrow u^{*}$, which is defined by

$$
\left(x_{1} \cdots x_{p}\right)^{*}=(-1)^{p} x_{p} \cdots x_{1}
$$

for $x_{1}, \ldots, x_{p} \in \mathfrak{g}$. If this antiautomorphism is extended to $U(\mathfrak{g}) \otimes U(\mathrm{~g})$ by $(u \otimes v)^{*}=v^{*} \otimes u^{*}$ then both the product $U(\mathrm{~g}) \otimes U(\mathrm{~g}) \rightarrow U(\mathrm{~g})$ and the diagonal map $U(\mathfrak{g}) \rightarrow U(\mathfrak{g}) \otimes U(\mathrm{~g})$ commute with the antiautomorphism.

The jet algebra $J\left(\mathrm{~g}^{*}\right)$ of a Lie algebra $\mathrm{g}$ is the dual space of the universal algebra $U(\mathrm{~g})$. The product on $J\left(\mathrm{~g}^{*}\right)$ is the map $J\left(\mathrm{~g}^{*}\right) \otimes J\left(\mathrm{~g}^{*}\right) \rightarrow J\left(\mathrm{~g}^{*}\right)$ dual to the diagonal map of $U(g)$, so

$$
(\alpha \beta, u)=(\alpha \otimes \beta, \Delta u)
$$

for $\alpha$ and $\beta$ in $J\left(g^{*}\right)$ and $u \in U(g)$. The associativity of the product follows from the associativity of the diagonal map. The unit of $J\left(g^{*}\right)$ is the augmentation of $U(\mathfrak{g})$. The q-jet algebra $J_{q}\left(\mathrm{~g}^{*}\right)$ of $\mathrm{g}$ is the dual space of $U_{q}(\mathrm{~g})$. The algebra structure of $J_{q}\left(g^{*}\right)$ is dual to the coalgebra structure of $U_{q}(g)$. Dual to the fact that $U(g)$ is the direct limit $U(\mathfrak{g})=\lim \operatorname{dir} U_{q}(\mathrm{~g})$, we have $J\left(\mathrm{~g}^{*}\right)=\lim \operatorname{inv} J_{q}\left(\mathrm{~g}^{*}\right)$. 
There is a natural pairing between $S^{q}\left(\mathrm{~g}^{*}\right)$ and $S^{q}(\mathrm{~g})$ defined by

$$
\left(\varphi_{1} \cdots \varphi_{q}, x_{1} \cdots x_{q}\right)=\sum_{\sigma} \varphi_{1}\left(x_{\sigma 1}\right) \cdots \varphi_{q}\left(x_{\sigma q}\right),
$$

the sum running over all permutations, and $S^{q}\left(\mathfrak{g}^{*}\right)$ may be identified with the dual of $S^{q}(\mathrm{~g})$. It follows that the dual of $S_{q}(\mathrm{~g})$ is $S_{q}\left(\mathrm{~g}^{*}\right)$ and that the dual of $S(\mathrm{~g})$ is the formal power series algebra $\hat{S}\left(\mathrm{~g}^{*}\right)$, the completion of $S\left(\mathrm{~g}^{*}\right)$. Moreover, it is not difficult to check that the pairing defined by (6) is a Hopf algebra pairing between $S\left(\mathrm{~g}^{*}\right)$ and $S(\mathrm{~g})$, i.e., that the product on $S\left(\mathrm{~g}^{*}\right)$ is dual to the diagonal map on $S(\mathrm{~g})$, and vice versa. We may consider $S_{q}\left(g^{*}\right)$ as the truncated polynomial algebra $S\left(\mathrm{~g}^{*}\right) / \sum_{p>q} S^{p}\left(\mathrm{~g}^{*}\right)$ and we have:

\section{Proposition 3.1. There are exact sequences}

$$
0 \longrightarrow S^{q}\left(\mathrm{~g}^{*}\right) \stackrel{i_{q}}{\longrightarrow} J_{q}\left(\mathrm{~g}^{*}\right) \stackrel{\pi_{q}}{\longrightarrow} J_{q-1}\left(\mathrm{~g}^{*}\right) \longrightarrow 0 .
$$

The q-jet algebra $J_{q}\left(\mathrm{~g}^{*}\right)$ is isomorphic to the truncated polynomial algebra $S_{q}\left(\mathrm{~g}^{*}\right)$, and the jet algebra $J\left(\mathrm{~g}^{*}\right)$ is isomorphic to the formal power series algebra $\hat{S}\left(\mathrm{~g}^{*}\right)$.

Proof. The proof is immediate by duality from the preceding remarks. The sequence (7) is dual to (2). The algebra isomorphism $\lambda_{q}^{*}: J_{q}\left(\mathrm{~g}^{*}\right) \rightarrow S_{q}\left(\mathrm{~g}^{*}\right)$ is the dual of the coalgebra isomorphism $\lambda_{q}: S_{q}(\mathrm{~g}) \rightarrow U_{q}(\mathrm{~g})$ and the algebra isomorphism $\lambda^{*}: J\left(\mathfrak{g}^{*}\right) \rightarrow \hat{S}\left(\mathrm{~g}^{*}\right)$ is the dual of the coalgebra isomorphism $\lambda: S(\mathfrak{g}) \rightarrow U(\mathfrak{g})$.

In particular, $J\left(\mathrm{~g}^{*}\right)$ is a commutative algebra. The antiautomorphism of $U(\mathfrak{g})$, commuting with the diagonal map, induces an automorphism $\alpha \rightarrow \alpha^{*}$ of $J\left(\mathfrak{g}^{*}\right)$ by

$$
\left(\alpha^{*}, u\right)=\left(\alpha, u^{*}\right)
$$

for $\alpha \in J\left(\mathrm{~g}^{*}\right)$ and $u \in U(\mathfrak{g})$.

By the universal property of $U(\mathfrak{g})$, a $\mathfrak{g}$-module is a module over the associative algebra $U(\mathrm{~g})$. We make the following conventions concerning representations: a simple $\mathfrak{g}$-module is a nonzero finite-dimensional $\mathfrak{g}$-module with no proper submodules, and a semisimple $\mathfrak{g}$-module is a direct sum of simple $\mathfrak{g}$-modules. Thus a semisimple $\mathfrak{g}$-module may be of infinite dimension, but it can have no irreducible components of infinite dimension.

If $M$ is a $g$-module then its dual $M^{*}$ is also, with

$$
(u \mu, m)=\left(\mu, u^{*} m\right)
$$

for $\mu \in M^{*}, m \in M$, and $u \in U(\mathfrak{g})$. If $M$ and $N$ are two $\mathfrak{g}$-modules then $M \otimes N$ is a $\mathfrak{g}$-module in three different ways. The representations may be called the left, the right, and the adjoint representations of $\mathfrak{g}$ on $M \otimes N$, and are defined by

and

$$
l(x)(m \otimes n)=x m \otimes n, \quad r(x)(m \otimes n)=m \otimes x n,
$$

$$
\theta(x)(m \otimes n)=x m \otimes n+m \otimes x n,
$$

for $x \in g, m \in M$, and $n \in N$. 
The universal algebra $U(\mathrm{~g})$ is also a $\mathrm{g}$-module under three different representations, defined by left multiplication, right multiplication, and the adjoint action of g. The dual representations on $J\left(g^{*}\right)$ are therefore given by

$$
(l(x) \alpha, u)=\left(\alpha, l\left(x^{*}\right) u\right)=\left(\alpha, x^{*} u\right), \quad(r(x) \alpha, u)=\left(\alpha, r\left(x^{*}\right) u\right)=(\alpha, u x),
$$

and

$$
(\theta(x) \alpha, u)=\left(\alpha, \theta\left(x^{*}\right) u\right)=(\alpha, u x-x u),
$$

for $x \in \mathfrak{g}, \alpha \in J\left(\mathrm{~g}^{*}\right)$, and $u \in U(\mathfrak{g})$.

Let now $\alpha \in J\left(\mathrm{~g}^{*}\right)$ and let $M(\alpha)$ be the submodule of $J\left(\mathrm{~g}^{*}\right)$ generated by $\alpha$ under the left representation of $\mathfrak{g}$ on $J\left(\mathrm{~g}^{*}\right)$. If $M(\alpha)$ is a semisimple $\mathfrak{g}$-module then $\alpha$ is called representative. If $\alpha$ is representative then $M(\alpha)$ must be finite-dimensional. For $M(\alpha)$ is a direct sum of finite-dimensional submodules, and $\alpha$ has a nonzero component in only finitely many of these. The submodule $M(\alpha)$ consists of the left translates $l(u) \alpha$ of $\alpha$, for all $u \in U(\mathrm{~g})$. Thus $\alpha$ is representative if and only if the left translates of $\alpha$ form a finite-dimensional semisimple submodule of $J\left(\mathrm{~g}^{*}\right)$ under the left action of $g$.

Let $R\left(\mathrm{~g}^{*}\right)$ be the subspace of representative elements of $J\left(\mathrm{~g}^{*}\right)$. If $\alpha \in R\left(\mathrm{~g}^{*}\right)$ then every element of $M(\alpha)$ is representative, so $M(\alpha)$ is a submodule of $R\left(g^{*}\right)$. Since $R\left(\mathrm{~g}^{*}\right)$ is the sum of the submodules $M(\alpha), \alpha \in R\left(\mathrm{~g}^{*}\right)$, it follows that it is the direct sum of some of them, and $R\left(\mathrm{~g}^{*}\right)$ is a semisimple submodule of $J\left(\mathrm{~g}^{*}\right)$ under the left action of $g$.

Let $\alpha$ and $\beta$ be any two elements of $J\left(\mathrm{~g}^{*}\right)$. The diagonal map $\Delta: U(\mathrm{~g}) \rightarrow U(\mathrm{~g})$ $\otimes U(\mathfrak{g})$ is an algebra homomorphism, so

$$
\begin{aligned}
(l(x)(\alpha \beta), u) & =\left(\alpha \otimes \beta, \Delta\left(x^{*} u\right)\right) \\
& =\left(\alpha \otimes \beta,\left(x^{*} \otimes 1+1 \otimes x^{*}\right) \Delta u\right) \\
& =(l(x) \alpha \cdot \beta+\alpha \cdot l(x) \beta, u)
\end{aligned}
$$

for $x \in \mathfrak{g}$ and $u \in U(\mathfrak{g})$. Thus $l(x)$ is a derivation of $J\left(\mathfrak{g}^{*}\right)$, and the multiplication $J\left(g^{*}\right) \otimes J\left(g^{*}\right) \rightarrow J\left(g^{*}\right)$ is a $g$-module homomorphism. If $\alpha$ and $\beta$ are representative the product $\alpha \beta$ lies in the image of the semisimple $g$-module $M(\alpha) \otimes M(\beta)$, and it follows that $\alpha \beta$ is representative. Thus $R\left(\mathrm{~g}^{*}\right)$ is a subalgebra of $J\left(\mathrm{~g}^{*}\right)$.

Let $M$ be a finite-dimensional semisimple $g$-module, and let $E(M)$ be the endomorphism algebra of $M$. The map dual to the representation $\rho: U(\mathrm{~g}) \rightarrow E(M)$ takes $E(M)^{*}$ into $J\left(\mathrm{~g}^{*}\right)$. We may identify $E(M)^{*}$ with $M \otimes M^{*}$, and with this identification the image of an element $m \otimes \mu$ of $M \otimes M^{*}$ in $J\left(\mathrm{~g}^{*}\right)$ is written $m / \mu$. Then $m / \mu$ is defined on $U(\mathrm{~g})$ by

$$
(m / \mu, u)=(u \mu, m)=\left(\mu, u^{*} m\right)
$$

for $u \in U(\mathfrak{g})$. It follows that $l(u)(m / \mu)=u m / \mu$, while $r(u)(m / \mu)=m / u \mu$. Each $\mu \in M^{*}$ therefore defines a $g$-module homomorphism $m \rightarrow m / \mu$, mapping $M$ onto a submodule of $J\left(\mathrm{~g}^{*}\right)$ invariant under the left action of $\mathrm{g}$. Since $M$ is semisimple, the elements $m / \mu$ are representative, and the image of $M \otimes M^{*} \rightarrow J\left(\mathrm{~g}^{*}\right)$ is contained 
in $R\left(\mathrm{~g}^{*}\right)$. For $\mu \in M^{*}$, let $/ \mu: M \rightarrow R\left(\mathrm{~g}^{*}\right)$ be the $\mathrm{g}$-module homomorphism defined by $\mu$.

LeMMA 3.2. For any finite-dimensional semisimple g-module $M$, the map $M^{*} \rightarrow \operatorname{Hom}_{\mathfrak{g}}\left(M, R\left(\mathrm{~g}^{*}\right)\right)$ given by $\mu \rightarrow / \mu$ is a vector space isomorphism.

Proof. Clearly $\mu \rightarrow \mid \mu$ is linear. Suppose $/ \mu=0$ for some $\mu \in M^{*}$. Then for every $m \in M,(\mu, m)=(m / \mu, 1)=0$. Thus $\mu=0$, and $\mu \rightarrow \mid \mu$ is $1-1$. Let $\varphi: M \rightarrow R\left(g^{*}\right)$ be any $\mathfrak{g}$-module homomorphism. Define $\mu \in M^{*}$ by $(\mu, m)=(\varphi(m), 1)$, for $m \in M$. Then $(m / \mu, u)=\left(\mu, u^{*} m\right)=\left(\varphi\left(u^{*} m\right), 1\right)=\left(u^{*} \varphi(m), 1\right)=(\varphi(m), u)$, and $/ \mu=\varphi$. Thus $\mu \rightarrow / \mu$ is onto.

Lemma 3.3. Suppose the field $F$ algebraically closed, and let $M$ be a simple $\mathrm{g}$-module. Then the map $M \otimes M^{*} \rightarrow R\left(\mathrm{~g}^{*}\right)$ is $1-1$.

Proof. This is clear from Burnside's Theorem, according to which the representation $\rho: U(\mathrm{~g}) \rightarrow E(M)$ is onto.

Let now $I$ index a complete family of inequivalent simple $g$-modules. For each $i \in I$ we have a canonical map from $M_{i} \otimes M_{i}^{*}$ into $R\left(\mathrm{~g}^{*}\right)$, and these maps define a map of the direct sum into $R\left(g^{*}\right)$.

THEOREM 3.4. If I indexes a complete family of inequivalent simple g-modules then the map

$$
\sum_{I} M_{i} \otimes M_{i}^{*} \rightarrow R\left(\mathrm{~g}^{*}\right)
$$

is onto, and commutes with the left, the right, and the adjoint action of $\mathrm{g}$. If the field $F$ is algebraically closed then (8) is an isomorphism.

Proof. By Lemma 3.2, every simple component of $R\left(\mathrm{~g}^{*}\right)$ isomorphic to one of the modules $M_{i}$ is in the image of $M_{i} \otimes M_{i}^{*}$. Since $R\left(\mathrm{~g}^{*}\right)$ is semisimple, and since the family indexed by $I$ is complete, the map (8) is onto. The relations $l(x)(m / \mu)=x m / \mu$ and $r(x)(m / \mu)=m / x \mu$ show that (8) commutes with the left and right action of $g$. Thus also $\theta(x)(m / \mu)=l(x)(m / \mu)+r(x)(m / \mu)=x m / \mu+m / x \mu$, and (8) commutes with the adjoint action of $\mathfrak{g}$. Finally, if $F$ is algebraically closed, then Lemma 3.3 shows that (8) is $1-1$ on each summand $M_{i} \otimes M_{i}^{*}$. Since the simple modules $M_{i}$ are inequivalent, and since (8) is a $g$-module homomorphism with respect to the left action of $g$, it must be injective. This completes the proof.

It follows from the theorem that $R\left(\mathrm{~g}^{*}\right)$ is closed under the automorphism $\alpha \rightarrow \alpha^{*}$ of $J\left(g^{*}\right)$. For every element of $R\left(g^{*}\right)$ may be written as a sum of elements of the form $m / \mu$, with $m$ an element of a simple g-module $M$, and $\mu$ in $M^{*}$. We have $(m / \mu)^{*}=\mu / m$, since $\left((m / \mu)^{*}, u\right)=\left(m / \mu, u^{*}\right)=(\mu, u m)=(\mu / m, u)$ for $u \in U(g)$. Since $M^{*}$ also is simple, $\mu / m$ is representative. The theorem also implies that the representative elements of $J\left(g^{*}\right)$ are precisely those which generate a semisimple submodule under the right action of $g$. 
Let now $M$ be any finite-dimensional semisimple $g$-module, and let $\rho: U(\mathfrak{g}) \rightarrow E(M)$ be the representation. The character of $M$ is the element $\chi_{M}$ of $J\left(g^{*}\right)$ defined by

$$
\left(\chi_{M}, u\right)=\operatorname{trace} \rho(u),
$$

for $u \in U(\mathrm{~g})$. If $m_{1}, \ldots, m_{r}$ is a basis of $M$, and $\mu_{1}, \ldots, \mu_{r}$ the dual basis of $M^{*}$ then

$$
\left(\chi_{M}, u\right)=\sum_{i}\left(\mu_{i}, u m_{i}\right)=\left(\sum_{i} \mu_{i} / m_{i}, u\right)
$$

and $\chi_{M}$ is representative. The relation $(m / \mu)^{*}=\mu / m$ implies that $\left(\chi_{M}\right)^{*}=\chi_{M}$. If $N$ is a second finite-dimensional semisimple $\mathrm{g}$-module then $\chi_{M}+\chi_{N}=\chi_{M \oplus N}$. Also, $\chi_{M} \chi_{N}=\chi_{M \otimes N}$. To see this, let $n_{1}, \ldots, n_{s}$ be a basis of $N$, and $\nu_{1}, \ldots, \nu_{s}$ be the dual basis of $N^{*}$. The elements $m_{i} \otimes n_{j}$ then form a basis of $M \otimes N$, and the elements $\mu_{i} \otimes \nu_{j}$ form the dual basis of $M^{*} \otimes N^{*}$. Given $u \in U(\mathrm{~g})$, let $\Delta u=\sum_{k} v_{k} \otimes w_{k}$. Using the fact that the adjoint, or tensor product representation of $U(\mathrm{~g})$ on $M \otimes N$ is defined in terms of the diagonal map, we have

$$
\begin{aligned}
\left(\chi_{M \otimes N}, u\right) & =\sum_{i, j}\left(\mu_{i} \otimes v_{j}, \theta(u)\left(m_{i} \otimes n_{j}\right)\right) \\
& =\sum_{i, j, k}\left(\mu_{i} \otimes v_{j}, v_{k} m_{i} \otimes w_{k} n_{j}\right) \\
& =\sum_{k}\left(\chi_{M} \otimes \chi_{N}, v_{k} \otimes w_{k}\right) \\
& =\left(\chi_{M} \chi_{N}, u\right),
\end{aligned}
$$

which proves the assertion.

Let $X\left(\mathrm{~g}^{*}\right)$ be the subspace of $R\left(\mathrm{~g}^{*}\right)$ generated by the characters of finite-dimensional semisimple $\mathrm{g}$-modules. We have shown that $X\left(\mathrm{~g}^{*}\right)$ is a subalgebra of $R\left(\mathrm{~g}^{*}\right)$.

Proposition 3.5. If I indexes a complete family of inequivalent simple g-modules $M_{i}$, and if $\chi_{i}$ is the character of $M_{i}$, then the family $\left(\chi_{i}\right)_{I}$ of characters is a basis of the vector space $X\left(\mathrm{~g}^{*}\right)$.

Proof. Since every finite-dimensional semisimple $g$-module $M$ is isomorphic to a direct sum of the simple $\mathfrak{g}$-modules $M_{i}$, each $M_{i}$ occurring with a multiplicity $n_{i}$, it follows that

$$
\chi_{M}=\sum_{I} n_{i} \chi_{i}
$$

and the $\chi_{i}$ span $X\left(\mathrm{~g}^{*}\right)$. That the $\chi_{i}$ are linearly independent is a consequence of Theorem 3.4, since $\chi_{i}$ is in the image of the submodule $M_{i} \otimes M_{i}^{*}$, a direct summand of $R\left(\mathrm{~g}^{*}\right)$.

By the proposition, the expression (9) is unique. Thus the multiplicities $n_{i}$, so $M$ itself, is uniquely determined by the character $\chi_{M}$ of $M$. 
Proposition 3.6. If the field $F$ is algebraically closed then $X\left(\mathrm{~g}^{*}\right)$ is the subalgebra of elements of $R\left(\mathrm{~g}^{*}\right)$ invariant under the adjoint representation of $\mathrm{g}$. With respect to the left or right representation of $\mathrm{g}, R\left(\mathrm{~g}^{*}\right)$ is generated by $X\left(\mathrm{~g}^{*}\right)$.

Proof. If $F$ is algebraically closed and if $M$ is a simple $g$-module then the elements of $E(M)^{*}$ invariant under the adjoint representation of $g$ are precisely the scalar multiples of the trace, by Schur's Lemma. This, with Theorem 3.4 and Proposition 3.5 , proves the first statement. To prove the second, note that the trace form $h \otimes k \rightarrow$ trace $h k$ is a nondegenerate bilinear form on $E(M)$. By Burnside's Theorem $\rho: U(\mathrm{~g}) \rightarrow E(M)$ is onto, so every element of $E(M)^{*}$ is of the form $k \rightarrow$ trace $\rho(u) k$, for some $u \in U(\mathrm{~g})$. This means that the character of $M$ generates the image of $M \otimes M^{*}$ in $R\left(\mathrm{~g}^{*}\right)$ under the left action of $\mathrm{g}$, so the second statement now follows from Theorem 3.4 .

4. Tensor and cotensor products. Let $\mathfrak{h}$ be a subalgebra of the Lie algebra $\mathfrak{g}$, and let $V$ be a finite-dimensional $\mathfrak{h}$-module. With respect to the right representation of $\mathfrak{h}$ on $U(\mathfrak{g})$ and the given representation of $\mathfrak{h}$ on $V, U(\mathfrak{g}) \otimes V$ is an $\mathfrak{h}$-module. The representation is defined by

$$
r_{v}(y)(u \otimes v)=r(y) u \otimes v+u \otimes y u=-u y \otimes v+u \otimes y v,
$$

for $y \in \mathfrak{h}, u \in U(\mathfrak{g})$, and $v \in V$. The quotient of $U(\mathfrak{g}) \otimes V$ by the subspace generated by all the elements (1) is the tensor product of $U(\mathfrak{g})$ and $V$ over $U(\mathfrak{h})$. We denote it by $U(\mathfrak{g}) \otimes_{\mathfrak{h}} V$. The operators $r_{v}(y), y \in \mathfrak{h}$, map $U_{q-1}(\mathfrak{g}) \otimes V$ into $U_{q}(\mathfrak{g}) \otimes V$. The quotient of $U_{q}(\mathfrak{g}) \otimes V$ by the subspace generated by the elements (1), with $y \in \mathfrak{h}$, $u \in U_{q-1}(\mathfrak{g})$, and $v \in V$, is called the tensor product of $U_{q}(\mathfrak{g})$ and $V$ over $\mathfrak{h}$, and is written $U_{q}(\mathfrak{g}) \otimes_{\mathfrak{h}} V$.

It is clear that the injection $U_{q-1}(\mathfrak{g}) \rightarrow U_{q}(\mathfrak{g})$ induces a map $U_{q-1}(\mathfrak{g}) \otimes_{\mathfrak{h}} V$ $\rightarrow U_{q}(\mathfrak{g}) \otimes_{\mathfrak{h}} V$. Let $\mathfrak{b}$ be the quotient space $\mathfrak{g} / \mathfrak{h}$. The natural map $\mathfrak{g} \rightarrow \mathfrak{b}$ extends to a homomorphism $S(\mathfrak{g}) \rightarrow S(\mathfrak{b})$ which vanishes on the ideal generated by $\mathfrak{h}$. On the other hand, the projection $U_{q}(\mathfrak{g}) \rightarrow S^{q}(\mathrm{~g})$ maps $U_{q-1}(\mathfrak{g})$ into zero, so the composition $U_{q}(\mathfrak{g}) \otimes V \rightarrow S^{q}(\mathfrak{g}) \otimes V \rightarrow S^{q}(\mathfrak{b}) \otimes V$ vanishes on the kernel of $U_{q}(\mathfrak{g}) \otimes V \rightarrow U_{q}(\mathfrak{g}) \otimes_{\mathfrak{h}} V$, and there is an induced map

$$
U_{q}(\mathfrak{g}) \otimes_{\mathfrak{h}} V \rightarrow S^{q}(\mathfrak{b}) \otimes V .
$$

Proposition 4.1. The sequence

$$
0 \rightarrow U_{q-1}(\mathfrak{g}) \otimes_{\mathfrak{h}} V \rightarrow U_{q}(\mathfrak{g}) \otimes_{\mathfrak{h}} V \rightarrow S^{q}(\mathfrak{b}) \otimes V \rightarrow 0
$$

is exact.

Proof. Choose a connection $\gamma: \mathfrak{b} \rightarrow \mathfrak{g}$, i.e., a linear map which composes with the natural map $\mathfrak{g} \rightarrow \mathfrak{b}$ to give the identity on $\mathfrak{b}$. Then $\gamma$ extends to a homomorphism $S(\mathfrak{b}) \rightarrow S(\mathfrak{g})$ which composes with the projection $S(\mathfrak{g}) \rightarrow S(\mathfrak{b})$ to give the identity 
on $S(\mathfrak{b})$. The composition of this homomorphism with the coalgebra isomorphism $\lambda: S(\mathfrak{g}) \rightarrow U(\mathfrak{g})$ yields maps $S_{q}(\mathfrak{b}) \otimes V \rightarrow U_{q}(\mathfrak{g}) \otimes V$, and we have a diagram

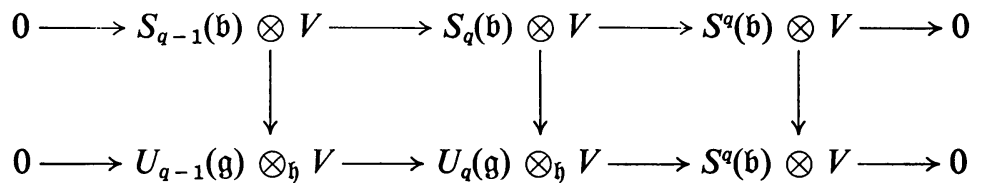

which is easily seen to be commutative. If $q=0$ then the map $S_{0}(\mathfrak{b}) \otimes V$ $\rightarrow U_{0}(\mathfrak{g}) \otimes_{\mathfrak{h}} V$ reduces to the identity on $V$. We may therefore suppose, inductively, that the map from $S_{q-1}(\mathfrak{b}) \otimes V$ to $U_{q-1}(\mathfrak{g}) \otimes_{\mathfrak{h}} V$ is an isomorphism. Every element of $U_{q}(g)$ may be written as a sum of elements of the form $z_{1} \cdots z_{r} y_{1} \cdots y_{s}$ with $z_{1}, \ldots, z_{r}$ in the image of $\gamma: \mathfrak{b} \rightarrow \mathfrak{g}$, and $y_{1}, \ldots, y_{s} \in \mathfrak{h}$. Thus, modulo elements of the form (1), every element of $U_{q}(g) \otimes V$ is congruent to a sum of elements of the form $z_{1} \cdots z_{r} \otimes v$, and it follows that the map $S_{q}(\mathfrak{b}) \otimes V \rightarrow U_{q}(\mathfrak{g}) \otimes_{\mathfrak{h}} V$ is onto. Since (3) is commutative, with an exact upper row, it follows that the lower row is also exact, and the proposition is proved.

Evidently the injection $U_{q}(\mathfrak{g}) \rightarrow U(\mathfrak{g})$ induces a map

$$
U_{q}(\mathfrak{g}) \otimes_{\mathfrak{h}} V \rightarrow U(\mathfrak{g}) \otimes_{\mathfrak{h}} V .
$$

Since $U(\mathfrak{g})$ is the union of the subspaces $U_{q}(\mathfrak{g})$, it follows that $U_{q}(\mathfrak{g}) \otimes_{\mathfrak{h}} V$ $\rightarrow U(\mathfrak{g}) \otimes_{\mathfrak{h}} V$ is injective. For if $P$ is an element of $U_{\mathfrak{q}}(\mathfrak{g}) \otimes_{\mathfrak{h}} V$ which is mapped into zero, then $P$ is also mapped into zero by $U_{q}(\mathfrak{g}) \otimes_{\mathfrak{h}} V \rightarrow U_{p}(\mathfrak{g}) \otimes_{\mathfrak{h}} V$ for some $p \geqq q$. By Proposition 4.1, this implies that $P=0$. Thus $U_{q}(\mathfrak{g}) \otimes_{\mathfrak{h}} V$ may be identified with a subspace of $U(\mathfrak{g}) \otimes_{\mathfrak{h}} V$, and $U(\mathfrak{g}) \otimes_{\mathfrak{h}} V$ is the union of these subspaces. The proof of Proposition 4.1 therefore shows that a connection $\gamma: \mathfrak{b} \rightarrow \mathfrak{g}$ defines an isomorphism $S(\mathfrak{b}) \otimes V \rightarrow U(\mathfrak{g}) \otimes_{\mathfrak{h}} V$.

An element $x \in \mathfrak{g}$ defines a left multiplication operator on $U(\mathfrak{g}) \otimes V$ by

$$
l(x)(u \otimes v)=x u \otimes v
$$

and it is clear that $l(x)$ commutes with the operators $r_{V}(y)$, for $y \in \mathfrak{h}$. Thus the quotient space $U(\mathfrak{g}) \otimes_{\mathfrak{h}} V$ is a $\mathfrak{g}$-module under the induced representation. On $U(\mathfrak{g}) \otimes_{\mathfrak{h}} V$, the operator $l(x)$ maps the subspace $U_{q}(\mathfrak{g}) \otimes_{\mathfrak{h}} V$ into $U_{q+1}(\mathfrak{g}) \otimes_{\mathfrak{h}} V$. For $y \in \mathfrak{h}$, let $\theta_{V}(y)$ be the operator on $U(\mathfrak{g}) \otimes V$ defined by

$$
\theta_{v}(y)(u \otimes v)=\theta(y) u \otimes v+u \otimes y t
$$

We have $\theta_{V}(y)(u \otimes v)=l(y)(u \otimes v)+r_{V}(y)(u \otimes v)$. It follows that there is an induced representation $\theta_{V}$ of $\mathfrak{h}$ on $U(\mathfrak{g}) \otimes_{\mathfrak{h}} V$, and that $\theta_{V}(y)=l(y)$ on $U(\mathfrak{g}) \otimes_{\mathfrak{h}} V$, for $y \in \mathfrak{h}$. The subspace $U_{q}(\mathfrak{g})$ of $U(\mathfrak{g})$ is stable under the adjoint representation of $\mathfrak{h}$, for we have

$$
\theta(y)\left(x_{1} \cdots x_{p}\right)=\sum_{i} x_{1} \cdots\left[y, x_{i}\right] \cdots x_{p}
$$


for $x_{1}, \ldots, x_{p} \in \mathfrak{g}$. It follows that the subspace $U_{q}(\mathrm{~g}) \otimes_{\mathfrak{h}} V$ of $U(\mathfrak{g}) \otimes_{\mathfrak{h}} V$ is stable under the representation $\theta_{V}$ of $\mathfrak{h}$. The adjoint representation of $\mathfrak{h}$ on $\mathfrak{g}$ preserves the subalgebra $\mathfrak{h}$, and there is an induced representation of $\mathfrak{h}$ on the quotient space $\mathfrak{b}$. The formula (4) serves to extend the adjoint representation to $S(\mathrm{~g})$, and it may similarly be extended to $S(\mathfrak{b})$. Tensoring with the given representation of $\mathfrak{h}$ on $V$, we obtain a representation $\theta_{V}$ of $\mathfrak{h}$ on $S(\mathfrak{b}) \otimes V$, and it follows easily that the sequence (2) is an exact sequence of $\mathfrak{h}$-modules and $\mathfrak{h}$-homomorphisms. A connection $\gamma: \mathfrak{b} \rightarrow \mathfrak{g}$ defines a map $S^{q}(\mathfrak{b}) \otimes V \rightarrow U_{q}(\mathfrak{g}) \otimes_{\mathfrak{h}} V$ which splits (2). Using the fact that $\lambda: S(\mathfrak{g}) \rightarrow U(\mathfrak{g})$ commutes with the adjoint action of $\mathfrak{h}$, it is not difficult to see that an invariant connection-one which commutes with the adjoint action of $\mathfrak{h}$-defines an $\mathfrak{h}$-invariant splitting of (2). Also, in this case, the isomorphism $S(\mathfrak{b}) \otimes V \rightarrow U(\mathfrak{g}) \otimes_{\mathfrak{h}} V$ is an $\mathfrak{h}$-module isomorphism.

Recall that the subalgebra $\mathfrak{h}$ of $\mathfrak{g}$ is reductive in $\mathfrak{g}$ if the adjoint representation of $\mathfrak{h}$ on $\mathfrak{g}$ is semisimple. The Lie algebra $\mathfrak{g}$ is reductive if it is reductive in itself. The universal algebra $U(\mathrm{~g})$ and the symmetric algebra $S(\mathrm{~g})$ are quotients of the tensor algebra $T(\mathfrak{g})$ of $\mathfrak{g}$. If $\mathfrak{h}$ is reductive in $\mathfrak{g}$ then $T(\mathfrak{g})$ is a semisimple $\mathfrak{h}$-module, so the same is true of $U(\mathfrak{g})$ and $S(\mathfrak{g})$. If $V$ is a semisimple $\mathfrak{h}$-module then so is $U(\mathfrak{g}) \otimes_{\mathfrak{h}} V$, a quotient of $U(\mathfrak{g}) \otimes V$. The submodules $U_{\mathfrak{q}}(\mathfrak{g}) \otimes_{\mathfrak{h}} V$ are therefore semisimple. Again, $\mathfrak{b}$ is semisimple, so $S(\mathfrak{b}) \otimes V$ and its submodules $S^{q}(\mathfrak{b}) \otimes V$ are semisimple. With $\mathfrak{h}$ reductive in $\mathfrak{g}$ an invariant connection $\gamma: \mathfrak{b} \rightarrow \mathfrak{g}$ always exists: it is defined by mapping $\mathfrak{b}$ onto a submodule of $\mathfrak{g}$ supplementary to $\mathfrak{h}$.

Now let $V^{*}$ be the dual of $V$, and consider $J\left(g^{*}\right) \otimes V^{*}$. Since $V$ is finite-dimensional, $J\left(\mathrm{~g}^{*}\right) \otimes V^{*}$ may be identified with the dual of $U(\mathrm{~g}) \otimes V$. A representation $r_{V^{*}}$ of $\mathfrak{h}$ is defined on the space $J\left(\mathfrak{g}^{*}\right) \otimes V^{*}$ by

$$
r_{V^{*}}(y)(\alpha \otimes \phi)=r(y) \alpha \otimes \phi+\alpha \otimes y \phi,
$$

and we have

$$
\left(r_{V^{*}}(y)(\alpha \otimes \phi), u \otimes v\right)=\left(\alpha \otimes \phi, r_{V}\left(y^{*}\right)(u \otimes v)\right)
$$

for $y \in \mathfrak{h}, \alpha \otimes \phi \in J\left(\mathfrak{g}^{*}\right) \otimes V^{*}$, and $u \otimes v \in U(\mathfrak{g}) \otimes V$. The subspace of elements of $J\left(g^{*}\right) \otimes V^{*}$ invariant under $r_{V}$. may therefore be identified with the dual of $U(\mathfrak{g}) \otimes_{\mathfrak{h}} V$. This subspace is called the cotensor product of $J\left(\mathfrak{g}^{*}\right)$ and $V^{*}$ over $\mathfrak{h}$, and is denoted by $J\left(\mathfrak{g}^{*}\right) \otimes^{\mathfrak{h}} V^{*}$. Interpreting now $\alpha \otimes \phi$ as an element of $J_{q}\left(\mathfrak{g}^{*}\right) \otimes V^{*}$, (5) defines a linear map

$$
r_{V^{*}}(y): J_{q}\left(g^{*}\right) \otimes V^{*} \rightarrow J_{q-1}\left(g^{*}\right) \otimes V^{*}
$$

It is then clear from (6) that the subspace of elements of $J_{q}\left(g^{*}\right) \otimes V^{*}$ annihilated by the maps $r_{V} \cdot(y), y \in \mathfrak{h}$, may be identified with the dual of $U_{q}(\mathfrak{g}) \otimes_{\mathfrak{h}} V$. This subspace is written $J_{q}\left(g^{*}\right) \otimes^{\mathfrak{h}} V^{*}$ and is called the cotensor product of $J_{q}\left(\mathfrak{g}^{*}\right)$ and $V^{*}$ over $\mathfrak{h}$. We may replace $V^{*}$ by $V$ in the definitions. The properties of the cotensor products $J\left(g^{*}\right) \otimes^{\mathfrak{h}} V$ and $J_{q}\left(g^{*}\right) \otimes^{\mathfrak{h}} V$ follow by duality. In particular, the proof of the following is clear. 
Proposition 4.2. The sequence

$$
0 \longrightarrow S^{q}\left(\mathfrak{b}^{*}\right) \otimes V \stackrel{i_{q}}{\longrightarrow} J_{q}\left(\mathfrak{g}^{*}\right) \otimes^{\mathfrak{h}} V \stackrel{\pi_{q}}{\longrightarrow} J_{q-1}\left(\mathfrak{g}^{*}\right) \otimes \mathfrak{h} V \longrightarrow 0
$$

is exact. The space $J_{q}\left(\mathrm{~g}^{*}\right) \otimes^{\mathfrak{h}} V$ may be identified with a quotient of $J\left(\mathrm{~g}^{*}\right) \otimes^{\mathfrak{h}} V$, and $J\left(\mathfrak{g}^{*}\right) \otimes^{\mathfrak{h}} V \simeq \lim \operatorname{inv} J_{q}\left(\mathfrak{g}^{*}\right) \otimes^{\mathfrak{h}} V$.

The space $J\left(\mathfrak{g}^{*}\right) \otimes^{\mathfrak{h}} V$ is a $\mathfrak{g}$-module under left action. This representation restricted to $\mathfrak{h}$ agrees with a representation $\theta_{V}$ defined by the adjoint action of $\mathfrak{h}$ on $J\left(\mathfrak{g}^{*}\right)$ and the given representation on $V$. The quotients $J_{q}\left(\mathfrak{g}^{*}\right) \otimes^{\mathfrak{h}} V$ are $\mathfrak{h}$-modules under $\theta_{V}$ and (7) is an exact sequence of $\mathfrak{h}$-modules and $\mathfrak{h}$-homomorphisms. An invariant connection $\gamma: \mathfrak{b} \rightarrow \mathfrak{g}$ defines an $\mathfrak{h}$-invariant splitting of (7). If $\mathfrak{h}$ is reductive in $\mathfrak{g}$ and if $V$ is a semisimple $\mathfrak{h}$-module, then $J_{q}\left(\mathfrak{g}^{*}\right) \otimes^{\mathfrak{h}} V$ is a semisimple $\mathfrak{h}$-module.

An element $\xi$ of $J\left(\mathrm{~g}^{*}\right) \otimes^{\xi} V$ is called representative if the submodule $M(\xi)$ generated by $\xi$ under the action of $g$ is semisimple. As before, it follows that $M(\xi)$ must then be finite-dimensional. Using the right action of $\mathfrak{h}$ on the subalgebra $R\left(\mathrm{~g}^{*}\right)$, the cotensor product $R\left(\mathrm{~g}^{*}\right) \otimes^{\mathfrak{h}} V$ is defined. Clearly, $R\left(\mathrm{~g}^{*}\right) \otimes^{\mathfrak{h}} V$ may be identified with a subspace of $J\left(\mathrm{~g}^{*}\right) \otimes^{\mathfrak{h}} V$.

Proposition 4.3. The representative elements of $J\left(\mathrm{~g}^{*}\right) \otimes^{\mathrm{h}} V$ are exactly the elements of $R\left(\mathfrak{g}^{*}\right) \otimes^{\mathfrak{h}} V$.

Proof. Let $v_{1}, \ldots, v_{n}$ be a basis of $V$. Every element $\xi$ of $J\left(\mathrm{~g}^{*}\right) \otimes^{\mathfrak{h}} V$ may then be uniquely written $\xi=\sum_{i} \alpha_{i} \otimes v_{i}$ for some $\alpha_{1}, \ldots, \alpha_{n}$ in $J\left(g^{*}\right)$. We have $l(u) \xi$ $=\sum_{i} l(u) \alpha_{i} \otimes v_{i}$ for $u \in U(\mathfrak{g})$, and it follows that $\xi$ is representative if and only if $\alpha_{1}, \ldots, \alpha_{n}$ are representative. This proves the proposition.

Let $M$ be a finite-dimensional semisimple $\mathfrak{g}$-module, hence also an $\mathfrak{h}$-module, by restriction. Given $m \otimes \zeta \in M \otimes \operatorname{Hom}_{\mathfrak{h}}(M, V)$, define

by

$$
m / \zeta \in J\left(g^{*}\right) \otimes V \simeq \operatorname{Hom}(U(g), V)
$$

$$
(m / \zeta)(u)=\zeta\left(u^{*} m\right)
$$

for $u \in U(\mathfrak{g})$. Then for $y \in \mathfrak{h},\left(r_{V}(y)(m / \zeta)\right)(u)=y((m / \zeta)(u))+(m / \zeta)(u y)=y\left(\zeta\left(u^{*} m\right)\right)$ $+\zeta\left(y^{*} u^{*} m\right)=0$, as $\zeta$ is $\mathfrak{h}$-linear. Thus $m / \zeta \in J\left(\mathfrak{g}^{*}\right) \otimes^{\mathfrak{h}} V$. Also, $l(x)(m / \zeta)=x m / \zeta$ for $x \in \mathfrak{g}$, and $m / \zeta$ is representative. The map $/ \zeta: M \rightarrow R\left(\mathfrak{g}^{*}\right) \otimes^{\mathfrak{h}} V$ defined by $m \rightarrow m / \zeta$ is a $g$-module homomorphism, and we have

Proposition 4.4 (ReCIPROCITY). For any finite-dimensional semisimple g-module $M$, the map $\zeta \rightarrow / \zeta$ is a vector space isomorphism

$$
\operatorname{Hom}_{\mathfrak{h}}(M, V) \rightarrow \operatorname{Hom}_{\mathfrak{g}}\left(M, R\left(\mathfrak{g}^{*}\right) \otimes^{\mathfrak{h}} V\right) .
$$

Proof. With $M^{*}$ replaced by $\operatorname{Hom}_{\mathfrak{h}}(M, V)$ and $R\left(\mathrm{~g}^{*}\right)$ replaced by $R\left(\mathrm{~g}^{*}\right) \otimes^{\mathfrak{h}} V$, the proof is that of Lemma 3.2. 
THEOREM 4.5. If I indexes a complete family of inequivalent simple g-modules, then the g-module homomorphism

$$
\sum_{I} M_{i} \otimes \operatorname{Hom}_{\mathfrak{h}}\left(M_{i}, V\right) \rightarrow R\left(\mathfrak{g}^{*}\right) \otimes^{\mathfrak{h}} V,
$$

given by $m \otimes \zeta \rightarrow m / \zeta$, is onto. If the field $F$ is algebraically closed, then (8) is an isomorphism.

Proof. The fact that (8) is onto follows from Proposition 4.4. If $F$ is algebraically closed then Theorem 3.4 shows that $(8)$ is $1-1$, for the diagram

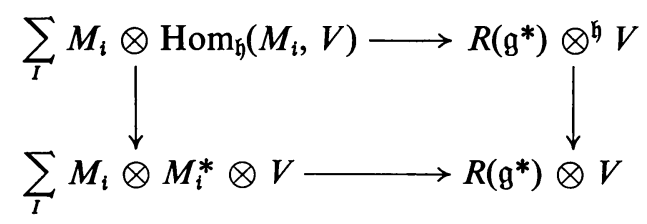

is commutative.

Let now $V$ and $W$ be two finite-dimensional $\mathfrak{h}$-modules. The cotensor product $J\left(\mathrm{~g}^{*}\right) \otimes^{\mathfrak{G}} V$ is filtered by subspaces $I_{q}(V)$, where $I_{q}(V)$ is the kernel of the projection $J\left(\mathrm{~g}^{*}\right) \otimes^{\mathfrak{h}} V \rightarrow J_{q}\left(\mathrm{~g}^{*}\right) \otimes^{\mathfrak{h}} V$. A linear map $D: J\left(\mathrm{~g}^{*}\right) \otimes^{\mathfrak{h}} V \rightarrow J\left(\mathrm{~g}^{*}\right) \otimes^{\mathfrak{h}} W$ is called a homomorphism, of order $r$, if $D$ maps $I_{q}(V)$ into $I_{q-r}(W)$ for every $q$. A homomorphism is invariant if it commutes with the action of $\mathrm{g}$. The invariant homomorphisms $D: J\left(\mathfrak{g}^{*}\right) \otimes^{\mathfrak{h}} V \rightarrow J\left(\mathrm{~g}^{*}\right) \otimes^{\mathfrak{h}} W$ form a vector space over $F$, filtered by order. Taking $V=W$, we get a filtered algebra.

It is clear that a homomorphism $D: J\left(\mathrm{~g}^{*}\right) \otimes^{\mathfrak{h}} V \rightarrow J\left(\mathrm{~g}^{*}\right) \otimes^{\mathfrak{h}} W$ of order $r$ induces linear maps $D_{q}$ and $D^{q}$ so that

(9)

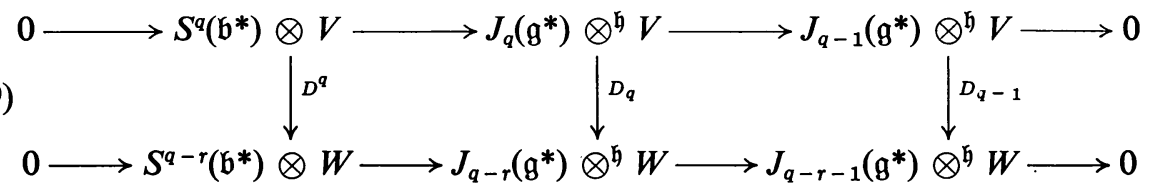

is commutative. If $D$ is invariant then it commutes with the action of $\mathfrak{h}$, and it follows easily that the maps of (9) are all $\mathfrak{h}$-module homomorphisms. We may identify $J_{0}\left(\mathrm{~g}^{*}\right) \otimes^{\mathfrak{h}} W$ with $W$, so an invariant homomorphism $D: J\left(\mathrm{~g}^{*}\right) \otimes^{\mathfrak{h}} V$ $\rightarrow J\left(\mathfrak{g}^{*}\right) \otimes^{\mathfrak{h}} W$ of order $r$ defines an element $D_{r} \in \operatorname{Hom}_{\mathfrak{h}}\left(J_{r}\left(\mathfrak{g}^{*}\right) \otimes^{\mathfrak{h}} V, W\right)$.

Proposition 4.6. The correspondence $D \rightarrow D_{r}$ is an isomorphism from the space of invariant homomorphisms $D: J\left(\mathrm{~g}^{*}\right) \otimes^{\mathfrak{h}} V \rightarrow J\left(\mathrm{~g}^{*}\right) \otimes^{\mathfrak{h}} W$ of order $r$, onto $\operatorname{Hom}_{\mathfrak{h}}\left(J_{r}\left(\mathfrak{g}^{*}\right) \otimes^{\mathfrak{h}} V, W\right)$.

Proof. We may identify $J\left(\mathfrak{g}^{*}\right) \otimes^{\mathfrak{h}} V$ with a subspace of $\operatorname{Hom}(U(\mathfrak{g}), V)$ and $J_{r}\left(g^{*}\right) \otimes^{h} V$ with a subspace of $\operatorname{Hom}\left(U_{r}(g), V\right)$. Let $\varphi_{r}$ be the projection $J\left(g^{*}\right) \otimes^{\mathfrak{h}} V$ $\rightarrow J_{r}\left(g^{*}\right) \otimes^{h} V$. Suppose that $D$ is an invariant homomorphism of order $r$. Then for $\xi \in J\left(g^{*}\right) \otimes^{\mathfrak{h}} V$ and $u \in U(\mathfrak{g}),(D \xi)(u)=\left(l\left(u^{*}\right) D \xi\right)(1)=\left(D l\left(u^{*}\right) \xi\right)(1)=\left(\varphi_{r} D l\left(u^{*}\right) \xi\right)(1)$ 
$=\left(D_{r} \varphi_{r} l\left(u^{*}\right) \xi\right)(1)=D_{r}\left(\varphi_{r} l\left(u^{*}\right) \xi\right)$. The last equality results from the identification of $J_{0}\left(g^{*}\right) \otimes^{h} W$ with $W$. It follows that $D_{r}=0$ only if $D=0$, so that $D \rightarrow D_{r}$ is injective. Now let $Q$ be any element of $\operatorname{Hom}_{\mathfrak{h}}\left(J_{r}\left(g^{*}\right) \otimes^{\mathfrak{h}} V, W\right)$ and define $D$, mapping $J\left(\mathfrak{g}^{*}\right) \otimes^{\mathfrak{h}} V$ into $J\left(\mathfrak{g}^{*}\right) \otimes W$, by

$$
(D \xi)(u)=Q\left(\varphi_{r} l\left(u^{*}\right) \xi\right) .
$$

We must show that $D \xi$ belongs to $J\left(g^{*}\right) \otimes^{\mathfrak{h}} W$. For $y \in \mathfrak{h}$,

$$
\left(r_{w}(y) D \xi\right)(u)=y((D \xi)(u))+(D \xi)(u y)
$$

By (10),

$$
\begin{aligned}
(D \xi)(u y) & =Q\left(\varphi_{r} l\left(y^{*}\right) l\left(u^{*}\right) \xi\right)=Q\left(\varphi_{r} \theta_{V}\left(y^{*}\right) l\left(u^{*}\right) \xi\right) \\
& =\theta_{\mathrm{w}}\left(y^{*}\right)\left(Q\left(\varphi_{r} l\left(u^{*}\right) \xi\right)=-y((D \xi)(u)) .\right.
\end{aligned}
$$

Thus $r_{w}(y) D \xi=0$, and $D \xi \in J\left(\mathfrak{g}^{*}\right) \otimes^{\mathfrak{h}} W$. Suppose $\xi \in I_{q}(V)$. Then for $u \in U_{q-r}(\mathrm{~g})$, $l\left(u^{*}\right) \xi$ is zero on $U_{r}(g)$. For if $v \in U_{r}(\mathfrak{g})$ we have $\left(l\left(u^{*}\right) \xi\right)(v)=\xi(u v)=\left(\varphi_{q} \xi\right)(u v)=0$. It follows that $\varphi_{r} l\left(u^{*}\right) \xi$ is zero, so $(D \xi)(u)=0$, by (10). This holds for $u \in U_{q-r}(\mathrm{~g})$, so $D \xi \in I_{q-r}(W)$. Thus $D: J\left(\mathfrak{g}^{*}\right) \otimes^{\mathfrak{h}} V \rightarrow J\left(\mathfrak{g}^{*}\right) \otimes^{\mathfrak{h}} W$ is a homomorphism of order $r$. For $x \in \mathfrak{g},(D l(x) \xi)(u)=Q\left(\varphi_{r} l\left(u^{*}\right) l(x) \xi\right)=Q\left(\varphi_{r} l\left(u^{*} x\right) \xi\right)=(D \xi)\left(x^{*} u\right)=(l(x) D \xi)(u)$, so $D$ is invariant. Evidently $D_{r}=Q$, so the proof is complete.

The proposition implies that the space of invariant homomorphisms of order $r$ may be identified with $\left(U_{r}(\mathfrak{g}) \otimes_{\mathfrak{h}} V^{*} \otimes W\right)^{\mathfrak{h}} \simeq\left(U_{r}(\mathfrak{g}) \otimes_{\mathfrak{h}} \operatorname{Hom}(V, W)\right)^{\mathfrak{h}}$. Note that the indicated tensor product is with respect to the right representation of $\mathfrak{h}$ on $\operatorname{Hom}(V, W)$, while $\left(U_{r}(\mathfrak{g}) \otimes_{\mathfrak{h}} \operatorname{Hom}(V, W)\right)^{\mathfrak{h}}$ indicates the subspace of invariants of $U_{r}(\mathfrak{g}) \otimes_{\mathfrak{h}} \operatorname{Hom}(V, W)$ with respect to the adjoint representation. The space of all invariant homomorphisms from $J\left(\mathfrak{g}^{*}\right) \otimes_{\mathfrak{h}} V$ to $J\left(\mathfrak{g}^{*}\right) \otimes_{\mathfrak{h}} W$ is isomorphic to $\left(U(\mathfrak{g}) \otimes_{\mathfrak{h}} \operatorname{Hom}(V, W)\right)^{\mathfrak{h}}$. If $\mathfrak{h}$ is reductive in $\mathfrak{g}$ and if $V$ and $W$ are semisimple $\mathfrak{h}$-modules, then the last space is a quotient of $(U(\mathfrak{g}) \otimes \operatorname{Hom}(V, W))^{\mathfrak{h}}$, itself a subspace of $U(\mathrm{~g}) \otimes \operatorname{Hom}(V, W)$. The semisimple nature of the module $U(\mathfrak{g})$ $\otimes \operatorname{Hom}(V, W)$ is needed to insure that the induced map

$$
(U(\mathfrak{g}) \otimes \operatorname{Hom}(V, W))^{\mathfrak{h}} \rightarrow\left(U(\mathfrak{g}) \otimes_{\mathfrak{h}} \operatorname{Hom}(V, W)\right)^{\mathfrak{h}}
$$

is onto. With this the case, however, every invariant homomorphism $D$ is represented by an element $\sum_{i} u_{i} \otimes \phi_{i}$ of $(U(\mathrm{~g}) \otimes \operatorname{Hom}(V, W))^{\mathfrak{h}}$, acting on

by

$$
\xi=\sum_{j} \alpha_{j} \otimes v_{j} \in J\left(\mathfrak{g}^{*}\right) \otimes^{\mathfrak{h}} V
$$

$$
D \xi=\sum_{i j} r\left(u_{i}\right) \alpha_{j} \otimes \phi_{i}\left(v_{j}\right)
$$

An invariant homomorphism $D: J\left(\mathfrak{g}^{*}\right) \otimes^{\mathfrak{h}} V \rightarrow J\left(\mathfrak{g}^{*}\right) \otimes^{\mathfrak{h}} W$ commutes with the action of $\mathrm{g}$, so maps $R\left(\mathrm{~g}^{*}\right) \otimes^{\mathfrak{h}} V$ into $R\left(\mathfrak{g}^{*}\right) \otimes^{\mathfrak{h}} W$. The restriction

$$
D: R\left(\mathrm{~g}^{*}\right) \otimes^{\mathfrak{h}} V \rightarrow R\left(\mathrm{~g}^{*}\right) \otimes^{\mathfrak{h}} W
$$


is homogeneous with respect to the direct sum decomposition defined by (8). If $K$ is the kernel of $D$ on $J\left(g^{*}\right) \otimes^{\mathfrak{h}} V$, then the kernel of $D$ on $R\left(\mathrm{~g}^{*}\right) \otimes^{\mathfrak{h}} V$ will be written $K_{R}$. Clearly, $K$ is closed under the action of $\mathfrak{g}$, and $K_{R}$ consists of the representative elements of $K$. With the notation of Theorem 4.5, if $K_{i}$ is the submodule which contains the elements of type $M_{i}$ of $K_{R}$, then $K_{i}$ is finite-dimensional and $K_{R}$ is the direct sum $\sum_{I} K_{i}$.

\section{Homogeneous Vector Bundles}

5. Lie groups. The field $F$ is again either the real or the complex field. Let $G$ be a Lie group, and let $\mathscr{F}(G)$ be the sheaf of germs of differentiable $F$-valued functions on $G$. An element $g \in G$ defines a left translation operator $l(g)$ on the sheaf $\mathscr{F}(G)$, mapping the stalk $\mathscr{F}(k)$ into the stalk $\mathscr{F}(g k)$, and given an $f \in \Gamma \mathscr{F}(G)$ by $(l(g) f)(k)=f\left(g^{-1} k\right)$. The right translation operator $r(g)$ defined by $g$ maps $\mathscr{F}(k)$ into $\mathscr{F}\left(k^{-1}\right)$, and is given on $\Gamma \mathscr{F}(G)$ by $(r(g) f)(k)=f(k g)$. Both $g \rightarrow l(g)$ and $g \rightarrow r(g)$ are representations of $G$ on the space $\Gamma \mathscr{F}(G)$. The automorphism $f \rightarrow f^{*}, f^{*}(k)=f\left(k^{-1}\right)$, interchanges the two representations. The adjoint representation of $G$ on $\Gamma \mathscr{F}(G)$ is defined by $\theta(g)=r(g) l(g)=l(g) r(g)$. The operators $\theta(g)$, acting on $\mathscr{F}(G)$, preserve the stalk $\mathscr{F}(e)$ at the identity.

Let $g$ be the Lie algebra of $G$. We identify $g$ with the tangent space at the identity, and define the bracket product to agree with the commutator of the corresponding left-invariant vector fields on $G$. By the conventions of $\$ 1, g$ depends on the field $F$, and is a complex Lie algebra if $F$ is the complex field. The left-invariant vector field defined by $x \in \mathfrak{g}$ is the infinitesimal right translation defined by $x$, and is denoted $r(x)$. Thus $x \rightarrow r(x)$ is the representation of $g$ on $\Gamma \mathscr{F}(G)$ corresponding to the group representation $g \rightarrow r(g)$. The Lie algebra representation corresponding to $g \rightarrow l(g)$ associates to $x \in \mathrm{g}$ a right-invariant vector field $l(x)$, related to $r(x)$ by $(l(x) f)^{*}=r(x) f^{*}$. While $r(x)$ has tangent vector $x$ at $e, l(x)$ has tangent vector $-x$. The operators $l(x)$ and $r(x)$ preserve the stalks of $\mathscr{F}(G)$, and $\mathscr{F}(k)$ is a g-module under the two representations, for $k \in G$. The adjoint operator defined by $x \in \mathfrak{g}$ is the sum $\theta(x)=l(x)+r(x)$.

The adjoint representation of $G$ on $g$ may be defined by $l(\theta(g) x)=\theta\left(g^{-1}\right) l(x) \theta(g)$ $=l\left(g^{-1}\right) l(x) l(g)$, or in terms of the left-invariant vector fields. The corresponding Lie algebra representation is the adjoint representation of $g$ on itself. The adjoint representation of $G$ is extended to $U(\mathrm{~g})$ by

$$
\theta(g)\left(x_{1} \cdots x_{p}\right)=\theta(g) x_{1} \cdots \theta(g) x_{p},
$$

and is defined on $J\left(\mathrm{~g}^{*}\right)$ by

$$
(\theta(g) \alpha, u)=\left(\alpha, \theta\left(g^{-1}\right) u\right) .
$$

Similarly, the adjoint representation of $G$ is defined on $S(\mathfrak{g})$ and $S\left(\mathrm{~g}^{*}\right)$. In all cases, the corresponding Lie algebra representation is the adjoint representation of $g$. 
As in $\S 1$, let $\mathscr{I}(k)$ be the ideal of $\mathscr{F}(k), k \in G$, consisting of the germs in $\mathscr{F}(k)$ which vanish at $k$. Clearly, the linear map $l(g): \mathscr{F}(k) \rightarrow \mathscr{F}(g k)$ maps $\mathscr{I}(k)$ into $\mathscr{I}(g k)$, hence maps $\mathscr{I}^{q}(k)$ into $\mathscr{I}^{q}(g k)$ for every $q$. Thus $l(g)$ induces an isomorphism $l(g): F_{q}(k) \rightarrow F_{q}(g k)$, given by

$$
l(g) j_{q, k}(\phi)=j_{q, g k}(l(g) \phi),
$$

for $\phi \in \mathscr{F}(k)$. The bundle $\boldsymbol{F}_{q}(G)$ is therefore determined by its fiber $\boldsymbol{F}_{q}(e)$ at the identity. This fiber is a $G$-module, with

$$
\theta(g) j_{q, e}(\phi)=j_{q, e}(\theta(g) \phi) .
$$

It follows from Lemma 1.1 that $l(x)$ and $r(x), x \in \mathfrak{g}$, are differential operators of order one on $\mathscr{F}(G)$. The associated bundle homomorphisms $\boldsymbol{F}_{q}(G) \rightarrow \boldsymbol{F}_{q-1}(G)$ are again written $l(x)$ and $r(x)$, so

$$
l(x) j_{q, e}(\phi)=j_{q-1, e}(l(x) \phi) \quad \text { and } \quad r(x) j_{q, e}(\phi)=j_{q-1, e}(r(x) \phi) .
$$

The adjoint operator $\theta(x)=l(x)+r(x)$ is also a differential operator of order one on $\mathscr{F}(G)$. However, the vector field $\theta(x)$ has tangent vector zero at the identity, so $\theta(x)$ maps $\mathscr{F}(e)$ into $\mathscr{F}(e)$. Since $\theta(x)$ is a derivation of $\mathscr{F}(e)$, it maps $\mathscr{I}^{q}(e)$ into itself for every $q$. Thus $\theta(x)$ induces an endomorphism of $\boldsymbol{F}_{q}(e)$,

$$
\theta(x) j_{q, e}(\phi)=j_{q, e}(\theta(x) \phi),
$$

and $F_{q}(e)$ is a g-module.

Define now a map $\Psi_{q}: F_{q}(e) \rightarrow J_{q}\left(g^{*}\right)$ by

$$
\left(\Psi_{q} j_{q, e}(\phi), u\right)=(r(u) \phi)(e)
$$

for $\phi \in \mathscr{F}(e)$ and $u \in U_{q}(g)$. The definition makes sense, for if $j_{q, e}(\phi)=0$, then $\phi \in \mathscr{I}^{q+1}(e)$ and for $u \in U_{q}(\mathfrak{g}), r(u) \phi$ is zero at $e$ by Lemma 1.1 .

Proposition 5.1. The map $\Psi_{q}: F_{q}(e) \rightarrow J_{q}\left(g^{*}\right)$ is an algebra isomorphism. The diagram

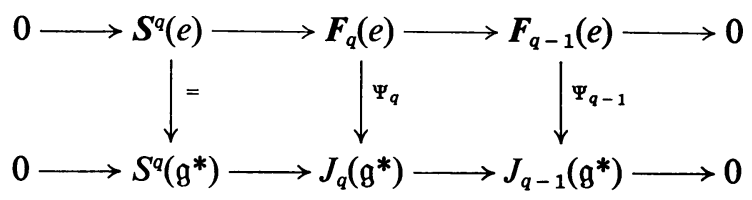

is commutative. The isomorphism $\Psi_{q}$ commutes with the adjoint representations of $G$ and $\mathrm{g}$, and we have $l(x) \Psi_{q}=\Psi_{q-1} l(x)$ and $r(x) \Psi_{q}=\Psi_{q-1} r(x)$ for $x \in \mathfrak{g}$.

Proof. We first show that (5) commutes. For $u \in U_{q-1}(g)$ we have

$$
\left(\Psi_{q} j_{q, e}(\phi), u\right)=(r(u) \phi)(e)=\left(\Psi_{q-1} j_{q-1, e}(\phi), u\right),
$$

which shows that the right-hand square commutes. Let $\phi_{1}, \ldots, \phi_{q}$ be elements of $\mathscr{I}(e)$. The composition $S^{q}(e) \rightarrow S^{q}\left(g^{*}\right) \rightarrow J_{q}\left(g^{*}\right)$ takes the product $d \phi_{1} \cdots d \phi_{q}$ into 
the linear map which vanishes on $U_{q-1}(\mathrm{~g})$ and is given on a product $x_{1} \cdots x_{q}$ of $q$ elements of $\mathfrak{g}$ by

$$
\sum_{\sigma} d \phi_{1}\left(x_{\sigma 1}\right) \cdots d \phi_{q}\left(x_{\sigma q}\right)
$$

the sum running over all permutations. The other composition takes $d \phi_{1} \cdots d \phi_{q}$ into $\Psi_{q} j_{q, e}\left(\phi_{1} \cdots \phi_{q}\right)$. If $u \in U_{q-1}(g)$ then $\left(\Psi_{q} j_{q, e}\left(\phi_{1} \cdots \phi_{q}\right), u\right)=\left(r(u)\left(\phi_{1} \cdots \phi_{q}\right)\right)(e)=0$, since $\phi_{1} \cdots \phi_{q}$ is an element of $\mathscr{I}^{q}(e)$. If $u=x_{1} \cdots x_{q}$, we use the fact that $r\left(x_{i}\right)$ is a derivation, and expand $\left(r(u)\left(\phi_{1} \cdots \phi_{q}\right)\right)(e)$ to get

$$
\sum_{\sigma}\left(r\left(x_{\sigma 1}\right) \phi_{1}\right)(e) \cdots\left(r\left(x_{\sigma q}\right) \phi_{q}\right)(e)=\sum_{\sigma} d \phi_{1}\left(x_{\sigma 1}\right) \cdots d \phi_{q}\left(x_{\sigma q}\right)
$$

again summed over all permutations. Thus (5) is commutative.

Since $j_{0, e}(\phi)=\phi(e), \Psi_{0}: F_{0}(e) \rightarrow J_{0}\left(g^{*}\right)$ reduces to the identity on $F$. Supposing, inductively, that $\Psi_{q-1}$ is a vector space isomorphism, the commutativity of (5) shows that $\Psi_{q}$ is a vector space isomorphism. Let $\phi$ and $\varphi$ be two elements of $\mathscr{F}(e)$ and let $u \in U_{q}(g)$. Suppose that $\Delta u=\sum_{i} v_{i} \otimes w_{i}$. Then

$$
\begin{aligned}
\left(\Psi_{q}\left(j_{q, e}(\phi) j_{q, e}(\varphi)\right), u\right) & =\left(\Psi_{q} j_{q, e}(\phi \varphi), u\right)=(r(u)(\phi \varphi))(e) \\
& =\sum_{i}\left(r\left(v_{i}\right) \phi\right)(e) \cdot\left(r\left(w_{i}\right) \varphi\right)(e)=\left(\Psi_{q} j_{q, e}(\phi) \otimes \Psi_{q} j_{q, e}(\varphi), \Delta u\right) \\
& =\left(\Psi_{q} j_{q, e}(\phi) \cdot \Psi_{q} j_{q, e}(\varphi), u\right),
\end{aligned}
$$

and $\Psi_{q}$ is an algebra isomorphism.

For $g \in G$,

$$
\begin{aligned}
\left(\theta(g) \Psi_{q} j_{q, e}(\phi), u\right) & =\left(\Psi_{q} j_{q, e}(\phi), \theta\left(g^{-1}\right) u\right)=\left(r\left(\theta\left(g^{-1}\right) u\right) \phi\right)(e)=\left(\theta\left(g^{-1}\right) r(u) \theta(g) \phi\right)(e) \\
& =(r(u) \theta(g) \phi)(e)=\left(\Psi_{q} j_{q, e}(\theta(g) \phi), u\right)=\left(\Psi_{q} \theta(g) j_{q, e}(\phi), u\right)
\end{aligned}
$$

using (1). Thus $\Psi_{q}$ is a $G$-module isomorphism. For $x \in \mathfrak{g}$,

But

$$
\begin{aligned}
\left(\theta(x) \Psi_{q} j_{q, e}(\phi), u\right) & =\left(\Psi_{q} j_{q, e}(\phi), \theta\left(x^{*}\right) u\right)=\left(r\left(\theta\left(x^{*}\right) u\right) \phi\right)(e) \\
& =(r(u x) \phi)(e)-(r(x u) \phi)(e) .
\end{aligned}
$$

$$
-(r(x u) \phi)(e)=-(r(x) r(u) \phi)(e)=(l(x) r(u) \phi)(e)=(r(u) l(x) \phi)(e),
$$

since right and left infinitesimal translations commute. Thus

$$
\begin{aligned}
(r(u x) \phi)(e)-(r(x u) \phi)(e) & =(r(u) \theta(x) \phi)(e)=\left(\Psi_{q} j_{q, e}(\theta(x) \phi), u\right) \\
& =\left(\Psi_{q} \theta(x) j_{q, e}(\phi), u\right),
\end{aligned}
$$

using (3), and it follows that $\Psi_{q}$ is a $\mathrm{g}$-module isomorphism. Similar computations, using (2), give $l(x) \Psi_{q}=\Psi_{q-1} l(x)$ and $r(x) \Psi_{q}=\Psi_{q-1} r(x)$, so the proof is complete.

We will use the isomorphism $\Psi_{q}$ to identify $\boldsymbol{F}_{q}(e)$ with $J_{q}\left(\mathrm{~g}^{*}\right)$. Then (4) becomes

$$
\left(j_{q, e}(\phi), u\right)=(r(u) \phi)(e)
$$


which defines $j_{q, e}(\phi), \phi \in \mathscr{F}(e)$, as an element of $J_{q}\left(g^{*}\right)$. The proposition implies that we may further identify the inverse limit $\boldsymbol{F}_{\infty}(e)$ with $J\left(\mathrm{~g}^{*}\right)$, and $j_{e}$ with a linear $\operatorname{map} j_{e}: \mathscr{F}(e) \rightarrow J\left(\mathrm{~g}^{*}\right)$ given by

$$
\left(j_{e}(\phi), u\right)=(r(u) \phi)(e)
$$

for $u \in U(\mathfrak{g})$. Then $j_{e}$ commutes with the adjoint representation of $G$, and with the left, right, and adjoint actions of $\mathfrak{g}$.

Let $\phi$ be an analytic element of $\mathscr{F}(k), k \in G$. Then $\phi$ is called representative if the submodule $M(\phi)$ of $\mathscr{F}(k)$, consisting of the infinitesimal left translates $l(u) \phi$ of $\phi$, is a semisimple $\mathfrak{g}$-module. Let $\mathscr{R}(k)$ contain the representative elements of $\mathscr{F}(k)$. Then $\mathscr{R}(k)$ is semisimple under the left action of $\mathfrak{g}$. Since $\mathfrak{g}$ operates on $\mathscr{R}(k)$ by derivations, it follows as before that $\mathscr{R}(k)$ is a subalgebra of $\mathscr{F}(k)$. The map $r(g): \mathscr{F}(k) \rightarrow \mathscr{F}\left(\mathrm{kg}^{-1}\right)$ defined by $g \in G$ evidently maps $\mathscr{R}(k)$ into $\mathscr{R}\left(\mathrm{kg}^{-1}\right)$.

Since $j_{e}: \mathscr{F}(e) \rightarrow J\left(\mathfrak{g}^{*}\right)$ commutes with the left action of $\mathfrak{g}$, it maps $\mathscr{R}(e)$ into $R\left(g^{*}\right)$.

Proposition 5.2. The restriction $j_{e}: \mathscr{R}(e) \rightarrow R\left(\mathrm{~g}^{*}\right)$ is an isomorphism.

Proof. Let exp be the exponential map, from a neighborhood of zero in $\mathrm{g}$ onto a neighborhood of $e$ in $G$. Let $\phi \in \mathscr{R}(e)$. Since $\phi$ is analytic, a function $f$ which represents $\phi$ is given in a neighborhood of $e$ by its Taylor expansion

$$
f(\exp x)=\sum_{p} \frac{1}{p !}\left(r\left(x^{p}\right) f\right)(e)=\sum_{p} \frac{1}{p !}\left(j_{e}(\phi), x^{p}\right) .
$$

If $j_{e}(\phi)=0$ then $f$ is zero in a neighborhood of $e$, and $\phi$ is zero. This shows that $j_{e}$ is $1-1$ on $\mathscr{R}(e)$.

By Theorem 3.4, every element of $R\left(\mathrm{~g}^{*}\right)$ is a sum of elements of the form $m / \mu$, where $m$ is an element of the simple $\mathfrak{g}$-module $M$, and $\mu \in M^{*}$. Let $\rho: U(\mathfrak{g}) \rightarrow E(M)$ be the representation, and write $\operatorname{Exp} x$ for the element $\sum_{p}(1 / p !) \rho(x)^{p}$ of $G L(M)$, $x \in \mathfrak{g}$. A function $f$ may be defined on a neighborhood of $e$ by

$$
f(\exp x)=((\operatorname{Exp} x) \mu, m)=\left(\mu,(\operatorname{Exp} x)^{-1} m\right) .
$$

Then $f$ is analytic, and since $\exp x \rightarrow \operatorname{Exp} x$ is a local homomorphism, we find that

$$
\begin{aligned}
(r(x) f)(\exp y) & =\lim _{t \rightarrow 0} \frac{f(\exp y \exp t x)-f(\exp y)}{t} \\
& =\lim _{t \rightarrow 0} \frac{\left((\operatorname{Exp} t x) \mu,(\operatorname{Exp} y)^{-1} m\right)-\left(\mu,(\operatorname{Exp} y)^{-1} m\right)}{t} \\
& =\left(x \mu,(\operatorname{Exp} y)^{-1} m\right) .
\end{aligned}
$$

Generally, then, $r(u) f$ is given by

$$
(r(u) f)(\exp y)=\left(u \mu,(\operatorname{Exp} y)^{-1} m\right) .
$$


Thus if $\phi \in \mathscr{F}(e)$ is the germ of $f$, then $\phi$ is representative, and

$$
\left(j_{e}(\phi), u\right)=(r(u) \phi)(e)=(r(u) f)(e)=(u \mu, m)=(m / \mu, u) .
$$

This shows that $j_{e}: \mathscr{R}(e) \rightarrow R\left(\mathrm{~g}^{*}\right)$ is onto, and completes the proof.

By the proposition, an element $m / \mu$ of $R\left(\mathrm{~g}^{*}\right)$ may be identified with a function defined in a neighborhood of $e$. Suppressing the notation for the exponential maps,

$$
(m / \mu)(g)=(g \mu, m)=\left(\mu, g^{-1} m\right),
$$

for $g$ near $e$. The proof gave $r(x)(m / \mu)=m / x \mu$ as the result of applying the leftinvariant vector field $r(x)$ to the function $m / \mu$. Similarly, the right-invariant vector field $l(x)$ is applied to $m / \mu$ by $l(x)(m / \mu)=x m / \mu$, so our notation is consistent. The function $m / \mu$ has a representative germ at every point of a neighborhood of $e$. An element $\phi \in \mathscr{R}(g)$ is a translate of an element of $\mathscr{R}(e)$, and it follows that $\mathscr{R}(G)=\bigcup_{G} \mathscr{R}(g)$ is a subsheaf of $\mathscr{F}(G)$. The global sections of $\mathscr{R}(G)$ are analytic functions on $G$, called representative functions.

Assume $G$ connected. Since the elements of $\Gamma \mathscr{R}(G)$ are analytic, the map $j_{e}: \Gamma \mathscr{R}(G) \rightarrow R\left(\mathrm{~g}^{*}\right)$ is $1-1$. Moreover, it is a $\mathrm{g}$-module homomorphism with respect to the left and right action of $\mathfrak{g}$. Thus $\Gamma \mathscr{R}(G)$ is a semisimple $\mathfrak{g}$-module under infinitesimal left or right translation. It is clear, however, that $\Gamma \mathscr{R}(G)$ is also a $G$-module under left and right translation, and the connectedness of $G$ implies that, with respect to either left or right action, the $G$-submodules and the g-submodules coincide. Let $I$ index a complete family of inequivalent simple $\mathfrak{g}$-modules. If the image of $j_{e}: \Gamma \mathscr{R}(G) \rightarrow R\left(\mathrm{~g}^{*}\right)$ meets the canonical image of $M_{i} \otimes M_{i}^{*}$ in $R\left(g^{*}\right)$ then it follows that the representation of $g$ on $M_{i}$ is induced by one of $G$. Conversely, if the representation of $g$ on $M_{i}$ is induced by one of $G$ then the local function $m / \mu$ given by $m \otimes \mu \in M_{i} \otimes M_{i}^{*}$ is defined on the whole of $G$ by (6), and it follows that the canonical image of $M_{i} \otimes M_{i}^{*}$ in $R\left(\mathrm{~g}^{*}\right)$ is contained in the image of $j_{e}: \Gamma \mathscr{R}(G) \rightarrow R\left(\mathrm{~g}^{*}\right)$. The map from $M_{i} \otimes M_{i}^{*}$ into $\Gamma \mathscr{R}(G)$ defined by (6) has the property that $l(g)(m / \mu)=g m / \mu$ and $r(g)(m / \mu)=m / g \mu$ for $g \in G$. These remarks, together with Theorem 3.4, prove:

Proposition 5.3. Let $G$ be connected. Let I index a complete family of inequivalent simple g-modules, and let $J$ index the subfamily for which the representation of $\mathrm{g}$ is induced by a representation of $G$. Then the map

$$
\sum_{J} M_{i} \otimes M_{i}^{*} \rightarrow \Gamma \mathscr{R}(G)
$$

given by $m \otimes \mu \rightarrow m / \mu$ is onto, and commutes with the left and right action of $G$; if $F$ is the complex field it is an isomorphism.

6. Homogeneous vector bundles. Let $H$ be a closed subgroup of $G$, and let $B=G / H$ be the homogeneous space of cosets $g H, g \in G$. We let $\eta: G \rightarrow B$ be the natural map, and call $o=\eta(e)$ the origin of $B$. The group $G$ acts naturally on $B$ by 
left translation, and $g \eta(k)=\eta(g k)$ for $g$ and $k$ in $G$. We identify the Lie algebra $\mathfrak{h}$ of $H$ with a subalgebra of $\mathfrak{g}$, and the tangent space to $B$ at the origin with $\mathfrak{g} / \mathfrak{h}=\mathfrak{b}$.

Let $V$ be a finite-dimensional $H$-module over $F$, and assume that the representation $H \rightarrow G L(V)$ is differentiable. To $V$ is then associated a differentiable vector bundle $V=G \times{ }_{H} V$ on $B$, the homogeneous vector bundle defined by $V$. An element of $V$ is an equivalence class of pairs $(g, v) \in G \times V$, with the equivalence relation defined by $(g, v) \sim\left(g h, h^{-1} v\right), h \in H$. The projection $V \rightarrow B$ is induced by the map $(g, v) \rightarrow \eta(g)$, and there is a commutative bundle diagram

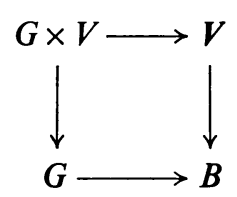

where $G \times V$ is regarded as the product bundle on $G$, and $G$ as a principal bundle on $B$. The action of $G$ on $V$ is induced by its action on $G \times V$, where $g(k, v)=(g k, v)$. If $\boldsymbol{V}(b)$ is the fiber of $\boldsymbol{V}$ at $b \in B$, then $g \in G$ defines a map $l(g): V(b) \rightarrow V(g b)$. In particular, $V(o)$ is an $H$-module under this action. The map $(e, v) \rightarrow v$ induces an $H$-module isomorphism $V(o) \rightarrow V$.

The correspondence between $H$-modules and homogeneous vector bundles has certain functorial properties. If $V$ and $W$ are two finite-dimensional $H$-modules then an $H$-module homomorphism $\varphi: V \rightarrow W$ gives rise to a bundle homomorphism $\Psi: V \rightarrow W$ which commutes with the action of $G$, induced by the map $(g, v)$ $\rightarrow(g, \varphi(v))$. This establishes a natural isomorphism

$$
\operatorname{Hom}_{H}(V, W) \rightarrow \operatorname{Hom}_{G}(\boldsymbol{V}, \boldsymbol{W})
$$

For if $\Psi: V \rightarrow W$ commutes with the action of $G$, then $\Psi(o): V(o) \rightarrow W(o)$ is an $H$-module homomorphism which defines $\varphi: V \rightarrow W$. In particular, the bundles $V$ and $W$ are $G$-isomorphic if and only if $V$ and $W$ are $H$-isomorphic. One may also check easily the following facts: the homogeneous vector bundle defined by $V \otimes W$ is $V \otimes W$, and that defined by $V^{*}$ is $V^{*}$. To an exact sequence of $H$ modules and $H$-homomorphisms is associated an exact sequence of homogeneous bundles and $G$-invariant bundle homomorphisms.

With the representation $H \rightarrow G L(V)$ differentiable, $V$ is also an $\mathfrak{h}$-module, and the cotensor product $J_{q}\left(\mathrm{~g}^{*}\right) \otimes^{\mathfrak{h}} V$ is defined. We saw in $\S 4$ that $J_{q}\left(\mathrm{~g}^{*}\right) \otimes^{\mathfrak{h}} V$ is again an $\mathfrak{h}$-module, under a representation $\theta_{V}$, defined by the adjoint representation of $\mathfrak{h}$ on $J_{q}\left(\mathfrak{g}^{*}\right)$ and the given representation on $V$. Correspondingly, $J_{q}\left(\mathfrak{g}^{*}\right) \otimes^{\mathfrak{h}} V$ is an $H$-module. This is clear if $H$ is connected, when the $\mathfrak{h}$-submodules and the $H$-submodules of $J_{q}\left(\mathrm{~g}^{*}\right) \otimes V$ agree, and it is not difficult to check directly for $H$ arbitrary. We set out now to find the homogeneous vector bundle defined by $J_{q}\left(\mathfrak{g}^{*}\right) \otimes^{\mathfrak{h}} V$. 
The bundle map $G \times V \rightarrow V$ is an isomorphism on each fiber, and, using (1), the differentiable sections of $V$ lift uniquely to differentiable functions $s: G \rightarrow V$, satisfying

$$
h(s(g))=s\left(g h^{-1}\right)
$$

for $g \in G$ and $h \in H$. The space of all differentiable functions $s: G \rightarrow V$ may be identified with $\Gamma \mathscr{F}(G) \otimes V$. Let $\mathscr{V}$ be the sheaf of germs of differentiable sections of $V$, as usual. The lifting process is an injection $\eta^{*}: \Gamma \mathscr{V} \rightarrow \Gamma \mathscr{F}(G) \otimes V$. The group $H$ operates on $\Gamma \mathscr{F}(G) \otimes V$ by

$$
r_{v}(h)(f \otimes v)=r(h) f \otimes h v,
$$

and the elements in the image of $\Gamma \mathscr{V}$, called the basic elements of $\Gamma \mathscr{F}(G) \otimes V$, are just the elements invariant under this representation. The group $G$, acting on $B$ and on $\boldsymbol{V}$, acts also on the sheaf $\mathscr{V}$, with $g \in G$ defining a linear map

$$
l(g): \mathscr{V}(b) \rightarrow \mathscr{V}(g b)
$$

for each $b \in B$. This makes $\Gamma \mathscr{V}$ into a $G$-module. Correspondingly, $\Gamma \mathscr{F}(G) \otimes V$ is a $G$-module, with

$$
l(g)(f \otimes v)=l(g) f \otimes v,
$$

and $\eta^{*}: \Gamma \mathscr{V} \rightarrow \Gamma \mathscr{F}(G) \otimes V$ is a $G$-module homomorphism. By adjoint action,

$$
\theta_{v}(h)(f \otimes v)=\theta(h) f \otimes h v,
$$

$\Gamma \mathscr{F}(G) \otimes V$ is also an $H$-module, and for $h \in H, l(h)$ and $\theta_{V}(h)$ agree on the basic elements of $\Gamma \mathscr{F}(G) \otimes V$.

Let $\mathscr{V}(o)$ be the stalk of $\mathscr{V}$ at the origin. In the obvious way, the injection $\eta^{*}: \Gamma \mathscr{V} \rightarrow \Gamma \mathscr{F}(G) \otimes V$ defines an injection $\eta^{*}: \mathscr{V}(o) \rightarrow \mathscr{F}(e) \otimes V$, mapping $\mathscr{V}(o)$ onto the subspace of basic elements of $\mathscr{F}(e) \otimes V$. For $h \in H, l(h)$ maps the stalk $\mathscr{V}(o)$ into itself, so $\mathscr{V}(o)$ is an $H$-module. Also, $\mathscr{F}(e) \otimes V$ is an $H$-module under a representation $\theta_{V}$ induced by (4), and the map $\eta^{*}: \mathscr{V}(o) \rightarrow \mathscr{F}(e) \otimes V$ is an $H$-module homomorphism.

Let $\boldsymbol{V}_{q}$ be the $q$-jet bundle of $\boldsymbol{V}$. The fiber $\boldsymbol{V}_{q}(b)$ at a point $b \in B$ is the quotient space $\mathscr{V}(b) / \mathscr{I}^{a+1}(b) \mathscr{V}(b)$. For $g \in G$, the map $l(g): \mathscr{F}(b) \rightarrow \mathscr{F}(g b)$ takes the ideal $\mathscr{I}(b)$ into $\mathscr{I}(g b)$, so $l(g): \mathscr{V}(b) \rightarrow \mathscr{V}(g b)$, commuting with the multiplication, takes $\mathscr{I}^{q+1}(b) \mathscr{V}(b)$ into $\mathscr{I}^{q+1}(g b) \mathscr{V}(g b)$ and induces a linear map $l(g): V_{q}(b) \rightarrow V_{q}(g b)$, given by

$$
l(g) j_{q, b}(\sigma)=j_{q, g b}(l(g) \sigma),
$$

for $\sigma \in \mathscr{V}(b)$. It follows that $V_{q}$ is a homogeneous vector bundle, and to identify $V_{q}$, it is necessary only to identify the $H$-module $V_{q}(o)$.

THEOREM 6.1. The homogeneous vector bundle associated to $J_{q}\left(\mathrm{~g}^{*}\right) \otimes^{\mathfrak{h}} V$ is the q-jet bundle $V_{q}$ of $V$. To the exact sequence

$$
0 \rightarrow S^{q}\left(\mathfrak{b}^{*}\right) \otimes V \rightarrow J_{q}\left(\mathfrak{g}^{*}\right) \otimes^{\mathfrak{h}} V \rightarrow J_{q-1}\left(\mathfrak{g}^{*}\right) \otimes^{\mathfrak{h}} V \rightarrow 0
$$


of H-modules and H-module homomorphisms is associated the exact sequence

$$
\mathbf{0} \rightarrow \boldsymbol{S}^{q} \otimes \boldsymbol{V} \rightarrow \boldsymbol{V}_{q} \rightarrow \boldsymbol{V}_{q-1} \rightarrow \mathbf{0}
$$

of homogeneous vector bundles and G-invariant bundle homomorphisms.

Proof. The map $\eta^{*}: \mathscr{F}(o) \rightarrow \mathscr{F}(e)$ takes $\mathscr{I}(o)$ into $\mathscr{I}(e)$. The $H$-module homomorphism $\eta^{*}: \mathscr{V}(o) \rightarrow \mathscr{F}(e) \otimes V$ commutes with multiplication by scalar germs, and takes $\mathscr{I}^{q+1}(o) \mathscr{V}(o)$ into the subspace $\mathscr{I}^{q+1}(e)(\mathscr{F}(e) \otimes V)=\mathscr{I}^{q+1}(e) \otimes V$, inducing a linear map $\eta^{*}: V_{q}(o) \rightarrow F_{q}(e) \otimes V$, again an $H$-module homomorphism. If for $\phi \otimes v \in \mathscr{F}(e) \otimes V$ we define $j_{q, e}(\phi \otimes v)=j_{q, e}(\phi) \otimes v$, then the induced map is given by

$$
\eta^{*} j_{q, b}(\sigma)=j_{q, e}\left(\eta^{*} \sigma\right)
$$

for $\sigma \in \mathscr{V}(o)$. Under the identification of $F_{q}(e)$ with $J_{q}\left(g^{*}\right), \eta^{*} j_{q, b}(\sigma)$ is an element of $J_{q}\left(\mathrm{~g}^{*}\right) \otimes V$. The Lie algebra representation corresponding to (2) is defined on $\mathscr{F}(e) \otimes V$ by

$$
r_{v}(y)(\phi \otimes v)=r(y) \phi \otimes v+\phi \otimes y v,
$$

where $r(y)$ is the left-invariant vector field on $G$ defined by $y \in \mathfrak{h}$. The basic elements of $\mathscr{F}(e) \otimes V$ are evidently annihilated by the operators $r_{V}(y)$, so by Proposition 5.1 we have $r_{V}(y) j_{q, e}\left(\eta^{*} \sigma\right)=j_{q-1, e}\left(r_{V}(y) \eta^{*} \sigma\right)=0$, and it follows that the induced map $\eta^{*}$ maps $V_{q}(o)$ into $J_{q}\left(\mathfrak{g}^{*}\right) \otimes^{\mathcal{H}} V$. It is easy to see that the map $\eta^{*}: V_{0}(o)$ $\rightarrow J_{0}\left(\mathrm{~g}^{*}\right) \otimes^{\mathfrak{h}} V$ reduces to the natural isomorphism $V(o) \rightarrow V$, so the proof will be complete by induction if we show that the diagram

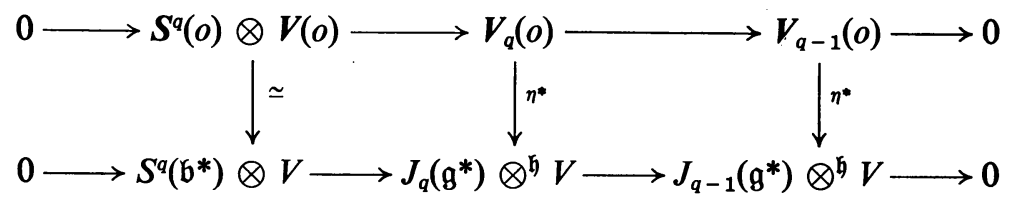

is commutative. The proof, however, is essentially the same argument used in the proof of Proposition 5.1.

The right-invariant vector fields $l(x)$ on $G$, operating on $\Gamma \mathscr{F}(G) \otimes V$ by

$$
l(x)(f \otimes v)=l(x) f \otimes v,
$$

preserve the basic elements of $\Gamma \mathscr{F}(G) \otimes V$, defining a representation of $\mathfrak{g}$ on $\Gamma \mathscr{V}$. For $f \in \Gamma \mathscr{F}(G)$ and $s \in \Gamma \mathscr{F}(G) \otimes V$,

$$
l(x)(f s)=l(x) f \cdot s+f \cdot l(x) s,
$$

so this relation also holds if $f$ is a differentiable function on $B$ and $s$ is an element of $\Gamma \mathscr{V}$. It follows easily that $l(x): \Gamma \mathscr{V} \rightarrow \Gamma \mathscr{V}$ is induced by a sheaf homomorphism $l(x)$ on $\mathscr{V}$. For if a section $s \in \Gamma \mathscr{V}$ is zero in a neighborhood of $b \in B$, let $f$ be a differentiable function, also zero in a neighborhood of $b$, and such that $f s=s$ on $B$. Then (7) implies that $l(x) s$ is zero in a neighborhood of $b$, so $l(x): \mathscr{V}(b) \rightarrow \mathscr{V}(b)$ is well defined. The relation (7) also shows that $l(x)$, operating on the differentiable 
functions on $B$, is a derivation, and it follows that $l(x)$ maps $\mathscr{I}^{q+1}(b) \mathscr{V}(b)$ into $\mathscr{I}^{q}(b) \mathscr{V}(b)$ for every $q$. Thus $l(x): \mathscr{V} \rightarrow \mathscr{V}$ is a differential operator of order one. An element $u \in U_{r}(\mathfrak{g})$ defines a differential operator $l(u)$ of order $r$ on $\mathscr{V}$. Each of the stalks of $\mathscr{V}$ is a $g$-module, and an analytic germ $\sigma \in \mathscr{V}(b)$ is called representative if the submodule $M(\sigma)$ of $\mathscr{V}(b)$ generated by $\sigma$ is semisimple. Let $\mathscr{V}_{R}(b)$ be the subspace of representative elements of $\mathscr{V}(b)$. For $g \in G$ and $u \in U(g)$ we have $l(g) l(u)=l(\theta(g) u) l(g)$, so $l(g): \mathscr{V}(b) \rightarrow \mathscr{V}(g b)$ maps $M(\sigma)$ into $M(l(g) \sigma)$ and $\mathscr{V}_{R}(b)$ into $\mathscr{V}_{R}(g b)$. The map $\eta^{*}: \mathscr{V}(o) \rightarrow \mathscr{F}(e) \otimes V$, commuting with the action of $\mathfrak{g}$, takes $\mathscr{V}_{R}(o)$ into $\mathscr{R}(e) \otimes V$. It follows easily that the composition $j_{e} \eta^{*}: \mathscr{V}(o)$ $\rightarrow J\left(\mathrm{~g}^{*}\right) \otimes^{\mathfrak{h}} V$ maps $\mathscr{V}_{R}(o)$ into $R\left(\mathrm{~g}^{*}\right) \otimes^{\mathfrak{h}} V$.

Proposition 6.2. The map $j_{e} \eta^{*}: \mathscr{V}_{R}(o) \rightarrow R\left(\mathrm{~g}^{*}\right) \otimes^{\mathfrak{h}} V$ is an isomorphism of g-modules. The subset $\mathscr{V}_{R}=\bigcup_{B} \mathscr{V}_{R}(b)$ is a subsheaf of $\mathscr{V}$.

Proof. The map $j_{e} \eta^{*}: \mathscr{V}_{R}(o) \rightarrow R\left(\mathrm{~g}^{*}\right) \otimes^{\mathfrak{h}} V$ is injective and is a $\mathrm{g}$-module homomorphism, since this is true of $\eta^{*}$, and also of $j_{e}$, by Proposition 5.2. By Theorem 4.5, every element of $R\left(\mathrm{~g}^{*}\right) \otimes^{\mathfrak{h}} V$ is a sum of those of the form $m / \zeta$, corresponding to $m \otimes \zeta$ in $M \otimes \operatorname{Hom}_{\mathfrak{b}}(M, V)$, with $M$ a simple $\mathfrak{g}$-module. As in Proposition 5.2, we may use the exponential map to define an analytic function in a neighborhood of $e$ in $G$ with values in $V$, by

$$
(m / \zeta)(g)=\zeta\left(g^{-1} m\right)
$$

Then $(m / \zeta)\left(g h^{-1}\right)=h((m / \zeta)(g))$ for $h$ and $g$ near $e$, and it follows that there exists a local analytic section $s$ of $V$, defined in a neighborhood of $o$, such that $j_{e} \eta^{*}(\sigma)=m / \zeta$, if $\sigma$ is the germ of $s$ at $o$. This shows that $j_{e} \eta^{*}$ is onto. Since $s$ has a representative germ at every point of a neighborhood of $o$, it follows by homogeneity that $\mathscr{V}_{R}$ is a subsheaf of $\mathscr{V}$, and the proof is complete.

If $G$ is connected then $B$ is connected and a section $s \in \Gamma \mathscr{V}_{R}$ is characterized by the fact that it is analytic and generates a semisimple submodule of $\Gamma \mathscr{V}$ under the action of $\mathfrak{g}$. Since $\eta^{*}: \Gamma \mathscr{V} \rightarrow \Gamma \mathscr{F}(G) \otimes V$ commutes with the action of $\mathfrak{g}$, it maps $\Gamma \mathscr{V}_{R}$ into $\Gamma \mathscr{R}(G) \otimes V$. Evidently the image of $\Gamma \mathscr{V}_{R}$ in $\Gamma \mathscr{R}(G) \otimes V$ consists of the basic elements of $\Gamma \mathscr{R}(G) \otimes V$, those invariant under the representation $r_{V}$ of $H$. Let $M$ be a simple $\mathfrak{g}$-module, and suppose the representation of $\mathfrak{g}$ is induced by one of $G$. Then for $m \otimes \zeta \in M \otimes \operatorname{Hom}_{H}(M, V)$, the function $m / \zeta: G \rightarrow V$,

$$
(m / \zeta)(g)=\zeta\left(g^{-1} m\right)
$$

is basic and belongs to $\Gamma \mathscr{R}(G) \otimes V$. We therefore have a map $M \otimes \operatorname{Hom}_{H}(M, V)$ $\rightarrow \Gamma \mathscr{V}_{R}$.

Proposition 6.3. Let $G$ and $H$ be connected. Let I index a complete family of inequivalent simple $\mathrm{g}$-modules, and let $J$ index the subfamily for which the representation of $\mathfrak{g}$ is induced by a representation of $G$. Then the map

$$
\sum_{J} M_{i} \otimes \operatorname{Hom}_{H}\left(M_{i}, V\right) \rightarrow \Gamma \mathscr{V}_{R}
$$

is onto, and a G-module homomorphism. If $F$ is the complex field, it is an isomorphism. 
Proof. Since $G$ is connected, $j_{e}: \Gamma \mathscr{R}(G) \otimes V \rightarrow R\left(\mathrm{~g}^{*}\right) \otimes V$ is injective. The image of a basic element is in $R\left(g^{*}\right) \otimes^{\mathfrak{h}} V$. Since the $g$-submodule generated by a basic element of $\Gamma \mathscr{R}(G) \otimes V$ is in fact a $G$-submodule, it follows from Theorem 4.5 that $j_{e}$ maps the basic elements of $\Gamma \mathscr{R}(G) \otimes V$ into the image of the direct sum $\sum_{J} M_{i} \otimes \operatorname{Hom}_{\mathfrak{G}}\left(M_{i}, V\right)$ in $R\left(\mathrm{~g}^{*}\right) \otimes^{\mathfrak{h}} V$. On the other hand, $j_{e}$ must map the basic elements of $\Gamma \mathscr{R}(G) \otimes V$ onto this image. For the connectedness of $H$ implies that $\operatorname{Hom}_{\mathfrak{h}}\left(M_{i}, V\right)=\operatorname{Hom}_{H}\left(M_{i}, V\right)$ for $i \in J$, while every element of

$$
M_{i} \otimes \operatorname{Hom}_{H}\left(M_{i}, V\right), \quad i \in J,
$$

defines a basic element of $\Gamma \mathscr{R}(G) \otimes V$, as remarked above. The proof is therefore clear.

7. Invariant differential operators. Let $V$ and $W$ be two finite-dimensional $\boldsymbol{H}$-modules, defined by differentiable representations. Let $\boldsymbol{V}$ and $\boldsymbol{W}$ be the associated homogeneous vector bundles on $B$, and let $\mathscr{V}$ and $\mathscr{W}$ be the corresponding sheaves. A differential operator $\mathscr{D}: \mathscr{V} \rightarrow \mathscr{W}$ is invariant if it commutes with the action of $\boldsymbol{G}$. Suppose that $\mathscr{D}$ is invariant and of order $r$, and let $\boldsymbol{D}_{q}: \boldsymbol{V}_{q} \rightarrow \boldsymbol{W}_{q-r}$ be the corresponding bundle homomorphism. According to Theorem 6.1, the bundles $\boldsymbol{V}_{\boldsymbol{q}}$ and $\boldsymbol{W}_{\boldsymbol{q}-\boldsymbol{r}}$ are homogeneous, and it now follows easily that $\boldsymbol{D}_{q}$ is an invariant bundle homomorphism. For if $b \in B$, if $\sigma \in \mathscr{V}(b)$, then $\boldsymbol{D}_{q} l(g) j_{q, b}(\sigma)=\boldsymbol{D}_{q} j_{q, g b}(l(g) \sigma)$ $=j_{q-r, g b}(\mathscr{D} l(g) \sigma)=j_{q-r, g b}(l(g) \mathscr{D} \sigma)=l(g) j_{q-r, b}(\mathscr{D} \sigma)=l(g) D_{q} j_{q, b}(\sigma)$ for $g \in G$. Conversely, let $D_{r}: V_{r} \rightarrow W$ be an invariant bundle homomorphism. By Theorem 2.2 there exists a unique differential operator $\mathscr{D}: \mathscr{V} \rightarrow \mathscr{W}$ of order $r$ inducing $D_{r}$, defined by $\mathscr{D} \sigma=D_{r} j_{r}(\sigma)$. Reversing the above computation shows that $\mathscr{D}$ is invariant. The correspondence $\mathscr{D} \rightarrow D_{r}$ is therefore 1-1 between invariant differential operators of order $r$ and invariant bundle homomorphisms. By Theorem 6.1, the invariant bundle homomorphisms $D_{r}: V_{r} \rightarrow W$ correspond uniquely to $H$-module homomorphisms $D_{r}: J_{r}\left(g^{*}\right) \otimes^{\mathfrak{h}} V \rightarrow W$. If $H$ is connected, then by Proposition 4.6 these last are in 1-1 correspondence with the $g$-invariant homomorphisms $D: J\left(g^{*}\right) \otimes^{\mathfrak{h}} V \rightarrow J\left(\mathfrak{g}^{*}\right) \otimes^{\mathfrak{h}} W$ of order $r$.

THEOREM 7.1. Let $H$ be connected. Then there is a natural isomorphism from the space of invariant homomorphisms

$$
D: J\left(\mathfrak{g}^{*}\right) \otimes^{\mathfrak{h}} V \rightarrow J\left(\mathfrak{g}^{*}\right) \otimes^{\mathfrak{h}} W
$$

to the space of invariant differential operators

$$
\mathscr{D}: \mathscr{V} \rightarrow \mathscr{W}
$$

If $D$ is of order $r$, then so is $\mathscr{D}$, and associated to the H-module homomorphisms

$$
\begin{gathered}
D_{q}: J_{q}\left(\mathfrak{g}^{*}\right) \otimes^{\mathfrak{h}} V \rightarrow J_{q-r}\left(\mathfrak{g}^{*}\right) \otimes^{\mathfrak{h}} W, \\
D^{q}: S^{q}\left(\mathfrak{b}^{*}\right) \otimes V \rightarrow S^{q-r}\left(\mathfrak{b}^{*}\right) \otimes W
\end{gathered}
$$


induced by $D$ are the bundle homomorphisms

induced by $\mathscr{D}$.

$$
\begin{gathered}
D_{q}: V_{q} \rightarrow W_{q-r}, \\
D^{q}: S^{q} \otimes V \rightarrow S^{q-r} \otimes W
\end{gathered}
$$

Proof. Given $\mathscr{D}$, the bundle homomorphism $\boldsymbol{D}_{q}$ is defined on the fiber $\boldsymbol{V}_{q}(o)$ by $\boldsymbol{D}_{q} j_{q, o}(\sigma)=j_{q-r, o}(\mathscr{D} \sigma), \sigma \in \mathscr{V}(o)$. The corresponding $H$-module homomorphism $D_{q}$ is then given by $D_{q} \eta^{*} j_{q, o}(\sigma)=\eta^{*} D_{q} j_{q, o}(\sigma)$, and $D$ is the inverse limit of the $D_{q}$. That the correspondence $\mathscr{D} \rightarrow D$ is an isomorphism has been proved. The $D_{q}$ are just the maps induced by $D$, so the proof of the second statement is also clear.

The relations $D_{q} \eta^{*} j_{q, o}(\sigma)=\eta^{*} D_{q} j_{q, o}(\sigma)=\eta^{*} j_{q-r, o}(\mathscr{D} \sigma)$ imply

$$
D_{q} j_{q, e}\left(\eta^{*} \sigma\right)=j_{q-r, e}\left(\eta^{*} \mathscr{D} \sigma\right),
$$

and, in the limit,

$$
D j_{e}(\eta * \sigma)=j_{e}(\eta * \mathscr{D} \sigma)
$$

for $\sigma \in \mathscr{V}(o)$. This relation and Theorem 7.1 continue to hold if $H$ is not connected, provided that the space of all invariant homomorphisms

$$
D: J\left(\mathfrak{g}^{*}\right) \otimes^{\mathfrak{h}} V \rightarrow J\left(\mathfrak{g}^{*}\right) \otimes^{\mathfrak{h}} W
$$

is replaced by the subspace of those which commute also with the action of $H$.

Let $\mathscr{D}: \mathscr{V} \rightarrow \mathscr{W}$ be an invariant differential operator, and let $D: J\left(g^{*}\right) \otimes^{\mathfrak{h}} V$ $\rightarrow J\left(g^{*}\right) \otimes^{\mathfrak{h}} W$ be the corresponding invariant homomorphism. Let $I$ index a complete family of inequivalent simple $g$-modules $M$. The kernel $K_{R}$ of $D$ restricted to $R\left(\mathrm{~g}^{*}\right) \otimes^{\mathfrak{h}} V$ is the direct sum of submodules $K_{i}$, with $K_{i}$ of type $M_{i}$. Assume $H$ connected. Then $K_{i}$, closed under the operators $l(y)=\theta_{v}(y), y \in \mathfrak{h}$, is also closed under the operators $\theta_{V}(h), h \in H$. Thus $K_{i}$ defines a homogeneous vector bundle $K_{i}$ on $B$.

Let $\mathscr{K}_{R}$ be the kernel of $\mathscr{D}$ restricted to $\mathscr{V}_{R}$. Since $\mathscr{D}$ is a sheaf homomorphism, $\mathscr{K}_{R}$ is a subsheaf of $\mathscr{V}_{R}$. Since $\mathscr{D}$ is invariant, $\mathscr{K}_{R}$ is closed under the action of $G$. It follows from (1) and Proposition 6.2 that $j_{e} \eta^{*}$ maps $\mathscr{K}_{R}(o)$ isomorphically onto $K_{R}$. Thus $\mathscr{K}_{R}(o)$ is closed under the action of $\mathfrak{g}$, and so is $\mathscr{K}_{R}(b)$, for any $b \in B$. It follows without difficulty that $\mathscr{K}_{R}$ is the direct sum of subsheaves $\mathscr{K}_{i}$, where $\mathscr{K}_{i}$ contains the elements of $\mathscr{K}_{R}$ which transform according to $M_{i}$. Each subsheaf $\mathscr{K}_{i}$ is closed under the action of $G$, and $j_{e} \eta^{*}: \mathscr{K}_{i}(o) \rightarrow K_{i}$ is an isomorphism of g-modules.

Given $b \in B$, the inverse limit $V_{\infty}(b)=\lim$ inv $V_{q}(b)$ is defined, and $j_{b}: \mathscr{V}(b)$ $\rightarrow \boldsymbol{V}_{\infty}(b)$ is defined as the inverse limit of the maps $j_{q, b}: \mathscr{V}(b) \rightarrow \boldsymbol{V}_{q}(b)$. The isomorphisms defined by translation, $l(g): V_{q}(b) \rightarrow V_{q}(g b)$, have as limit an isomorphism $l(g): V_{\infty}(b) \rightarrow V_{\infty}(g b)$, and $l(g) j_{b}(\sigma)=j_{g b}(l(g) \sigma)$ for $\sigma \in \mathscr{V}(b)$. The limit of the isomorphisms $\eta^{*}: V_{q}(o) \rightarrow J_{q}\left(g^{*}\right) \otimes^{\mathfrak{h}} V$ is an isomorphism $\eta^{*}: V_{\infty}(o) \rightarrow J\left(g^{*}\right) \otimes^{\mathfrak{h}} V$, and $j_{e} \eta^{*}=\eta^{*} j_{o}$ on $\mathscr{V}(o)$. This leads us to identify the fiber $K_{i}(o)$ with the image in 
$V_{\infty}(o)$ of $j_{o}: \mathscr{K}_{i}(o) \rightarrow V_{\infty}(o)$. Then $j_{0}: \mathscr{K}_{i}(o) \rightarrow K_{i}(o)$ is a $g$-module isomorphism. Using again the fact that $H$ is connected, it is an $H$-module isomorphism, and we may identify $\boldsymbol{K}_{i}(b)$ with the image in $\boldsymbol{V}_{\infty}(b)$ of $j_{b}: \mathscr{K}_{i}(b) \rightarrow \boldsymbol{V}_{\infty}(b)$, for any $b \in B$. Then the isomorphisms $j_{b}: \mathscr{K}_{i}(b) \rightarrow K_{i}(b)$ commute with the action of $\mathfrak{g}$, and the relation $l(g) j_{b}(\sigma)=j_{g b}(l(g) \sigma), \sigma \in \mathscr{K}_{i}(b)$, continues to hold.

Let $s$ be a local section of the sheaf $\mathscr{K}_{i}$. Define a local section $j(s)$ of the bundle $K_{i}$ by $j(s)(b)=j_{b}(s)$, if $s$ is defined in a neighborhood of $b \in B$. On $G$, where the bundles are trivial, we have

LEMMA 7.2. Given $\sum_{j} m_{j} / \zeta_{j} \in K_{i}$, let $s$ be defined in a neighborhood of $e$, with values in $V$, by $s(g)=\sum_{j} \zeta_{j}\left(g^{-1} m_{j}\right)$. Then $j(s)$, defined in a neighborhood of $e$, and with values in $K_{i}$, is given by $j(s)(g)=g^{-1}\left(\sum_{j} m_{j} / \zeta_{j}\right)=\sum_{j}\left(g^{-1} m_{j}\right) / \zeta_{j}$.

Proof. We have $j(s)(g)=j_{g}(s)=j_{e}\left(l\left(g^{-1}\right) s\right)$. But $l\left(g^{-1}\right) s$ is given by the element $\sum_{j}\left(g^{-1} m_{j}\right) / \zeta_{j}$ of $K_{i}$, so $j_{e}\left(l\left(g^{-1}\right) s\right)=\sum_{j}\left(g^{-1} m_{j}\right) / \zeta_{j}$.

From the relation $j_{e} \eta^{*}=\eta^{*} j_{o}$, it follows that $j \eta^{*}=\eta^{*} j$ on local sections of $\mathscr{K}_{i}$, since both sides of the last relation commute with the action of $G$. Thus if $s$ is a local section of $\mathscr{K}_{i}$ defined in a neighborhood of $o$, it follows from the lemma that $j(s)$ is a differentiable section of $K_{i}$. Let $\mathscr{K}_{i}^{0}$ be the sheaf of germs of differentiable sections of $\boldsymbol{K}_{\boldsymbol{i}}$. We have proved:

Proposition 7.3. The map $j$ is a sheaf homomorphism $j: \mathscr{K}_{i} \rightarrow \mathscr{K}_{i}^{0}$. The sequence

is exact.

$$
0 \longrightarrow \mathscr{K}_{i} \stackrel{j}{\longrightarrow} \mathscr{K}_{i}^{0}
$$

The second statement is obvious, since $j_{b}$ is an isomorphism, for each $b \in B$. We note also that $j$ commutes with the action of $G$ on the sheaves. For $x \in \mathfrak{g}$ the relation $j \cdot l(x)=l(x) \cdot j$ holds, provided that, on $\mathscr{K}_{i}^{0}, l(x)$ is interpreted as the extension of the bundle homomorphism $l(x): \boldsymbol{K}_{i} \rightarrow \boldsymbol{K}_{i}$.

\section{Cоноmology}

8. Lie algebra cohomology. Let $\mathfrak{g}$ be a Lie algebra over a field $F$ of characteristic zero, and let $M$ be any $g$-module. Let $g^{*}$ be the dual of $g$, and let $\Lambda\left(g^{*}\right)$ be the exterior algebra of $\mathfrak{g}^{*}$. Standardly, $\Lambda\left(\mathfrak{g}^{*}\right) \otimes M$ is made into a complex in the following way. The adjoint representation of $g$ on $\Lambda\left(g^{*}\right)$ is combined with the given representation of $\mathfrak{g}$ on $M$, making $\Lambda\left(\mathfrak{g}^{*}\right) \otimes M$ into a $\mathfrak{g}$-module. This defines an operator $\theta_{M}(x)$ of degree zero on $\Lambda\left(\mathfrak{g}^{*}\right) \otimes M$, for $x \in \mathfrak{g}$. By interior $\mathrm{n}_{\text {. }}$ ultiplication, $x \in \mathfrak{g}$ also defines an operator $\mathrm{t}(x)$ of degree -1 on $\Lambda\left(\mathrm{g}^{*}\right) \otimes M$, and the relations

$$
\iota(x) \iota(y)+\iota(y) \iota(x)=0
$$

and

$$
\theta_{M}(x) \iota(y)-\iota(y) \theta_{M}(x)=\iota([x, y])
$$


hold for $x$ and $y$ in $\mathfrak{g}$. There exists a unique operator $d_{M}$ on $\Lambda\left(\mathfrak{g}^{*}\right) \otimes M$, of degree +1 , such that

$$
\iota(x) d_{M}+d_{M^{\iota}}(x)=\theta_{M}(x)
$$

for all $x \in \mathfrak{g}$. One proves that

$$
\theta_{M}(x) d_{M}=d_{M} \theta_{M}(x)
$$

for $x \in \mathfrak{g}$, and finally, that $d_{M}^{2}=0$.

Let $\mathfrak{h}$ be a subalgebra of $\mathfrak{g}$. From (3) and (4) it follows that the elements of $\Lambda\left(\mathrm{g}^{*}\right) \otimes M$ annihilated by the operators $\iota(y)$ and $\theta_{M}(y), y \in \mathfrak{h}$, form a subcomplex $C\left(\mathfrak{g}^{*}, \mathfrak{h}^{*}, M\right)$. As a vector space, $C\left(\mathfrak{g}^{*}, \mathfrak{h}^{*}, M\right)$ is seen to be naturally isomorphic to the subspace $\left(\Lambda\left(\mathfrak{b}^{*}\right) \otimes M\right)^{\mathfrak{h}}$ of invariants of $\Lambda\left(\mathfrak{b}^{*}\right) \otimes M$. Here, $\mathfrak{b}$ is the quotient $\mathfrak{g} / \mathfrak{h}$, as usual, and $\Lambda\left(\mathfrak{b}^{*}\right) \otimes M$ is an $\mathfrak{h}$-module via the adjoint representation on $\Lambda\left(\mathfrak{b}^{*}\right)$, and the restriction to $\mathfrak{h}$ of the representation of $\mathfrak{g}$ on $M$. The cohomology of the complex $C\left(\mathfrak{g}^{*}, \mathfrak{h}^{*}, M\right)$ is the relative cohomology with coefficients in $M$, written $H\left(\mathfrak{g}^{*}, \mathfrak{h}^{*}, M\right)$. If $M$ is the trivial $\mathfrak{g}$-module $F$, we write $H\left(\mathfrak{g}^{*}, \mathfrak{b}^{*}\right)$ for the relative cohomology.

Lemma 8.1. Let $\mathrm{g}$ be reductive and let $M$ be a simple nontrivial $\mathrm{g}$-module. Then there exists an operator $c$ on $\Lambda\left(\mathrm{g}^{*}\right) \otimes M$, of degree -1 , such that $C=c d_{M}+d_{M} c$ is an automorphism of $\Lambda\left(\mathrm{g}^{*}\right) \otimes M$, while $\iota(x) c+c \iota(x)=0$ and $\theta_{M}(x) c=c \theta_{M}(x)$ for $x \in \mathfrak{g}$.

Proof. This is well known if $\mathfrak{g}$ is semisimple. In any case, $\mathfrak{g}$ is the direct sum of its center $\mathfrak{c}$, and its derived ideal $\mathfrak{b}$, which is semisimple. If there exists an element $z \in \mathfrak{c}$ which is not represented by zero on $M$, take $c=\iota(z)$. Then $C=\theta_{M}(z)$ acts as $1 \otimes z$ on $\Lambda\left(\mathrm{g}^{*}\right) \otimes M$, which is an automorphism. The remaining properties of $c$ follow from (1) and (2). If $\mathrm{c}$ is in the kernel of the representation then $M$ is a simple $\mathfrak{D}$-module, and the usual proof applies.

Proposition 8.2. Let $\mathrm{g}$ be reductive, and let $M$ be a semisimple g-module. Then $H\left(\mathfrak{g}^{*}, \mathfrak{h}^{*}, M\right) \simeq H\left(\mathfrak{g}^{*}, \mathfrak{h}^{*}\right) \otimes M^{g}$.

Proof. Suppose first that $M$ is simple and nontrivial. The lemma implies that the subcomplex $\left.C\left(\mathfrak{g}^{*}, \mathfrak{h}\right)^{*}, M\right)$ is closed under $c$ and $C$. If $\omega \in C\left(\mathfrak{g}^{*}, \mathfrak{h}^{*}, M\right)$ is a cocycle then $C \omega=d_{M} c \omega$ is a coboundary, and so is $\omega=C^{-1} d_{M} c \omega=d_{M} C^{-1} c \omega$. Thus $H\left(\mathfrak{g}^{*}, \mathfrak{h}^{*}, M\right)$ is zero. In the general case, $M$ is a direct sum $\sum_{\mathfrak{i}} M_{\mathfrak{i}}$ of simple $\mathrm{g}$-modules, and there are corresponding decompositions

and

$$
C\left(\mathfrak{g}^{*}, \mathfrak{h}^{*}, M\right) \simeq \sum_{i} C\left(\mathfrak{g}^{*}, \mathfrak{h}^{*}, M_{i}\right)
$$

$$
H\left(\mathfrak{g}^{*}, \mathfrak{h}^{*}, M\right) \simeq \sum_{i} H\left(\mathfrak{g}^{*}, \mathfrak{h}^{*}, M_{i}\right) .
$$

The first part of the proof now applies to give the conclusion. 
9. The resolution. The purpose of this section is to prove the following theorem:

THEOREM 9.1. Let $H$ be connected. Let $\mathscr{D}: \mathscr{V} \rightarrow \mathscr{W}$ be an invariant differential operator, and let $\mathscr{K}_{i}$ be the sheaf of representative elements of type $M_{i}$ in its kernel. Let $D: J\left(\mathfrak{g}^{*}\right) \otimes^{\mathfrak{h}} V \rightarrow J\left(\mathfrak{g}^{*}\right) \otimes^{\mathfrak{h}} W$ be the corresponding invariant homomorphism, with $K_{i}$ the submodule of representative elements of type $M_{i}$ in its kernel. Let $\mathscr{K}_{i}^{p}$ be the sheaf of germs of differential p-forms with values in the homogeneous bundle defined by $K_{i}$. Then there exists an invariant differential operator $d_{i}$ of order one, such that

$$
0 \longrightarrow \mathscr{K}_{i} \stackrel{j}{\longrightarrow} \mathscr{K}_{i}^{0} \stackrel{d_{i}}{\longrightarrow} \mathscr{K}_{i}^{1} \stackrel{d_{i}}{\longrightarrow} \cdots
$$

is a resolution.

Proof. The initial terms of the resolution are described in Proposition 7.3. We proceed now to construct $d_{i}$. On the space $\Gamma \mathscr{F}(G) \otimes K_{i}$, define a representation $r_{i}$ of $g$ by

$$
r_{i}(x)(f \otimes \xi)=r(x) f \otimes \xi+f \otimes l(x) \xi .
$$

We are assuming that $H$ is connected, so the basic elements of $\Gamma \mathscr{F}(G) \otimes K_{i}$ are just the elements annihilated by the operators $r_{i}(y), y \in \mathfrak{h}$. Following the procedure of the previous section, we may define a representation $\theta_{i}$ of $\mathfrak{g}$ on $\Lambda\left(\mathrm{g}^{*}\right)$ $\otimes \Gamma \mathscr{F}(G) \otimes K_{i}$ by combining the adjoint representation on $\Lambda\left(g^{*}\right)$ with the representation $r_{i}$. There exists then a unique differential $d_{i}$ on this complex such that $\iota(x) d_{i}+d_{i} \iota(x)=\theta_{i}(x)$ for $x \in \mathfrak{g}$. The elements of the relative complex

$$
C\left(\mathfrak{g}^{*}, \mathfrak{h}^{*}, \Gamma \mathscr{F}(G) \otimes K_{\mathfrak{i}}\right)
$$

may evidently be identified with the basic elements of the space $\Gamma \mathscr{F}(G)$ $\otimes \Lambda\left(\mathfrak{b}^{*}\right) \otimes K_{i}$, which correspond, in turn, to the differentiable sections of the homogeneous bundle defined by $\Lambda\left(\mathfrak{b}^{*}\right) \otimes K_{i}$. In other words, if $K_{i}$ is the homogeneous bundle defined by $K_{i}$, then $C\left(\mathfrak{g}^{*}, \mathfrak{h}^{*}, \Gamma \mathscr{F}(G) \otimes K_{i}\right)$ is isomorphic to the space of differential forms on $B$ with values in $\boldsymbol{K}_{\boldsymbol{i}}$.

The complex $\Lambda\left(\mathrm{g}^{*}\right) \otimes \Gamma \mathscr{F}(G)$ is the usual complex of differential forms on $G$, and the differential is the exterior differential $d$. In general, $\Lambda\left(\mathrm{g}^{*}\right) \otimes \Gamma \mathscr{F}(G) \otimes K_{i}$ is a module over $\Lambda\left(\mathrm{g}^{*}\right) \otimes \Gamma \mathscr{F}(G)$, and

$$
d_{i}(\Phi \wedge \Omega)=d \Phi \wedge \Omega+(-1)^{p} \Phi \wedge d_{i} \Omega
$$

if $\Phi$ is a scalar differential $p$-form, and $\Omega$ a differential form with values in $K_{i}$. Similar expressions are valid with $d_{i}$ replaced by $\iota(x)$ or $\theta_{i}(x), x \in \mathfrak{g}$. Thus if $\Phi$ and $\Omega$ are basic then so is $\Phi \wedge \Omega$, and we may proceed as in $\S 6$ to reason that $d_{i}$ is a differential operator. First, if $\Omega$ is zero in a neighborhood of $b \in B$, we may choose $f$, also zero in a neighborhood of $b$, and such that $f \Omega=\Omega$. Then (2) shows that $d_{i}(f \Omega)$ is zero in a neighborhood of $b$, so $d_{i}$ is a sheaf homomorphism. Since the 
exterior differential is a derivation, $d_{i}$ maps $\mathscr{I}^{q+1}(b) \mathscr{K}_{i}^{p}(b)$ into $\mathscr{I}^{q}(b) \mathscr{K}_{i}^{p+1}(b)$, and $d_{i}$ is a differential operator of order one. Finally, since $\mathfrak{g}$ acts on $\Gamma \mathscr{F}(G) \otimes K_{i}$ by operators $r_{i}(x)$, the operators on the complex $\Lambda\left(g^{*}\right) \otimes \Gamma \mathscr{F}(G) \otimes K_{i}$ commute with left translation by $G$, and $d_{i}$ is an invariant differential operator.

We have now constructed the sequence (1), and it remains to show that it is a resolution. Since the sheaf homomorphisms $j$ and $d_{i}$ commute with the action of $G$, it will be sufficient to show that the lower row of the diagram

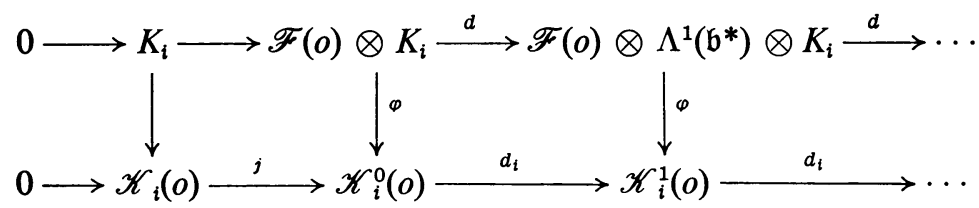

is exact. For this purpose, we define a second representation of $\mathfrak{g}$ on $\Gamma \mathscr{F}(G) \otimes K_{i}$, by

$$
r(x)(f \otimes \xi)=r(x) f \otimes \xi .
$$

The corresponding differential is the exterior differential tensored with the identity of $K_{i}$, and this, on the basic germs in $\Lambda\left(\mathfrak{b}^{*}\right) \otimes \mathscr{F}(e) \otimes K_{i}$, gives the upper row of (3). By the ordinary Poincaré Lemma, the upper row is exact. Define now a map $\varphi: \mathscr{F}(e) \otimes K_{i} \rightarrow \mathscr{F}(e) \otimes K_{i}$ as follows. Since $K_{i}$ is a $g$-module, it is a local $G$ module, and if $f$ is defined in a neighborhood of $e$, we may define $\varphi(f \otimes \xi)$ by

$$
\varphi(f \otimes \xi)(g)=f(g)\left(g^{-1} \xi\right),
$$

inducing a map $\varphi: \mathscr{F}(e) \otimes K_{i} \rightarrow \mathscr{F}(e) \otimes K_{i}$. For $g$ and $k$ near $e, \varphi(r(g) f \otimes \xi)(k)$ $=f(k g)\left(k^{-1} \xi\right)=g\left(f(k g)\left(g^{-1} k^{-1} \xi\right)\right)=r_{i}(g) \varphi(f \otimes \xi)(k)$, and it follows that $\varphi r(x)$ $=r_{i}(x) \varphi$ on $\mathscr{F}(e) \otimes K_{i}$, for $x \in \mathfrak{g}$. It follows that $\varphi$ induces an isomorphism of complexes, and we have defined the maps of (3), with the exception of the map $K_{i} \rightarrow \mathscr{K}_{i}(o)$. This map associates to $\xi=\sum_{j} m_{j} / \zeta_{j}$ the germ of the local section corresponding to the basic function $s$, where $s(g)=\sum_{j} \zeta_{j}\left(g^{-1} m_{j}\right)$. It is an isomorphism, and by Lemma 7.2, it completes (3) to a commutative diagram. The lower row of (3) is therefore exact, and the proof of the theorem is complete.

The sheaves $\mathscr{K}_{i}^{p}$ are fine, so the de Rham theorem applies, and the cohomology of $B$ with coefficients in the sheaf $\mathscr{K}_{i}$ is that of the complex of global differential forms on $B$ with values in the bundle $K_{i}$, with the differential $d_{i}$. In other words,

COROLlaRY. The cohomology of $B$ with coefficients in the sheaf $\mathscr{K}_{i}$ is isomorphic to $H\left(\mathfrak{g}^{*}, \mathfrak{h}^{*}, \Gamma \mathscr{F}(G) \otimes K_{i}\right)$.

10. Compact groups. Suppose now that $G$ is compact. Then $B$ is compact, and it follows that the cohomology of $B$ with coefficients in $\mathscr{K}_{i}$ is finite-dimensional. For there exists a neighborhood $U$ of $o$, depending only on the exponential map, such that each of the elements of $\mathscr{K}_{i}(o)$ is the germ of an analytic section of $\mathscr{V}$ defined on $U$. We can suppose that $U$ is connected. Then the restriction map 
$\Gamma \mathscr{K}_{i}(U) \rightarrow \mathscr{K}_{i}(b)$ is an isomorphism, for any $b \in U$. Let $\mathscr{U}$ be a finite covering of $B$ made up of translates of $U$. It follows easily that the cohomology group defined by the covering $\mathscr{U}$, clearly finite-dimensional, is isomorphic to that of $B$ with coefficients in $\mathscr{K}_{i}$. Thus, with $H$ connected, the corollary to Theorem 9.1 implies that $H\left(\mathfrak{g}^{*}, \mathfrak{h}^{*}, \Gamma \mathscr{F}(G) \otimes K_{\mathfrak{i}}\right)$ is finite-dimensional.

Let now $J$ index the subfamily of those simple $g$-modules $M_{j}$ which are also $G$-modules. Suppose the field $F$ is the complex field. Then the map

$$
\sum_{J} M_{j} \otimes \operatorname{Hom}\left(M_{j}, K_{i}\right) \rightarrow \Gamma \mathscr{F}(G) \otimes K_{i}
$$

defined by $m \otimes \zeta \rightarrow m / \zeta$ is injective and commutes with the left action of $G$. With $g$ acting on $\operatorname{Hom}\left(M_{j}, K_{i}\right)$ via the adjoint representation and on $\Gamma \mathscr{F}(G) \otimes K_{i}$. via the representation $r_{i}$ of the last section, (1) is a $g$-module homomorphism. It follows that there is an induced map on cohomology

$$
\sum_{J} H\left(\mathfrak{g}^{*}, \mathfrak{h}^{*}, M_{j} \otimes \operatorname{Hom}\left(M_{j}, K_{i}\right)\right) \rightarrow H\left(\mathfrak{g}^{*}, \mathfrak{h}^{*}, \Gamma \mathscr{F}(G) \otimes K_{i}\right) .
$$

Since the action of $G$ commutes with the differentials, the cohomology groups are $G$-modules, and (2) is a $G$-module homomorphism. We intend to show that it is an isomorphism.

Because $G$ is compact, the Haar integral is defined on $\Gamma \mathscr{F}(G)$ and may be extended to a linear function

$$
\int: \Gamma \mathscr{F}(G) \otimes K_{i} \rightarrow K_{i}
$$

Given $f \in \Gamma \mathscr{F}(G)$ and $s \in \Gamma \mathscr{F}(G) \otimes K_{i}$, we may define a convolution product $f * s \in \Gamma \mathscr{F}(G) \otimes K_{i}$ by

$$
(f * s)(g)=\int\left(f^{*}\right) r(g) s
$$

and it follows easily that $l(g)(f * s)=l(g) f * s$ and $r(g)(f * s)=f * r(g) s$, using the invariance of the integral. Corresponding relations hold for the operation of $\mathfrak{g}$, and we have

$$
r_{i}(x)(f * s)=f * r_{i}(x) s
$$

for $x \in \mathfrak{g}$. For $j \in J$, let $\chi_{j}$ be the character of $M_{j}$, considered as a function on $G$, and set $\varepsilon_{j}=\left(\operatorname{dim} M_{j}\right) \chi_{j}$. As in the usual case, it follows that the map $s \rightarrow \varepsilon_{j} * s$ is a projection from $\Gamma \mathscr{F}(G) \otimes K_{i}$ onto the image of $M_{j} \otimes \operatorname{Hom}\left(M_{j}, K_{i}\right)$ in $\Gamma \mathscr{F}(G) \otimes K_{i}$. By (3), it commutes with the operation of $\mathfrak{g}$, hence extends to a homomorphism of complexes. If we assume $G$ connected, it follows from Proposition 6.3 that the image of

$$
C\left(\mathfrak{g}^{*}, \mathfrak{h}^{*}, M_{j} \otimes \operatorname{Hom}\left(M_{j}, K_{i}\right)\right) \simeq M_{j} \otimes \operatorname{Hom}_{\mathfrak{h}}\left(M_{j}, \Lambda\left(\mathfrak{b}^{*}\right) \otimes K_{\mathfrak{i}}\right)
$$

in $C\left(\mathfrak{g}^{*}, \mathfrak{h}^{*}, \Gamma \mathscr{F}(G) \otimes K_{i}\right)$ is just the image of the projection $\Omega \rightarrow \varepsilon_{j} * \Omega$, and consists of those elements transforming according to $M_{j}$ under the action of $G$. 
Similar statements hold, therefore, for the cocycle and coboundary groups, and finally, for the cohomology groups. Thus (2) maps the component

$$
H\left(\mathfrak{g}^{*}, \mathfrak{h}^{*}, M_{j} \otimes \operatorname{Hom}\left(M_{j}, K_{i}\right)\right)
$$

isomorphically onto the direct summand consisting of those elements which transform according to $M_{j}$. But $G$ is compact, and since $H\left(\mathfrak{g}^{*}, \mathfrak{h}^{*}, \Gamma \mathscr{F}(G) \otimes K_{\mathfrak{i}}\right)$ is a finite-dimensional $G$-module, it is semisimple. Thus (2) is an isomorphism.

It is clear that $\left.H\left(\mathfrak{g}^{*}, \mathfrak{h}\right)^{*}, M_{j} \otimes \operatorname{Hom}\left(M_{j}, K_{i}\right)\right)$ is isomorphic to

$$
M_{j} \otimes H\left(\mathfrak{g}^{*}, \mathfrak{h}^{*}, \operatorname{Hom}\left(M_{j}, K_{\mathfrak{i}}\right)\right) \text {. }
$$

Since $G$ is compact, $\mathfrak{g}$ is reductive, and Proposition 8.2 applies. It follows that we have $H\left(\mathfrak{g}^{*}, \mathfrak{h}^{*}, \operatorname{Hom}\left(M_{j}, K_{i}\right)\right) \simeq H\left(\mathfrak{g}^{*}, \mathfrak{h}^{*}\right) \otimes \operatorname{Hom}_{\mathfrak{g}}\left(M_{j}, K_{i}\right)$. For $j \neq i, \operatorname{Hom}_{\mathfrak{g}}\left(M_{j}, K_{i}\right)$ is zero, while $M_{j} \otimes \operatorname{Hom}_{\mathfrak{g}}\left(M_{j}, K_{j}\right) \simeq K_{j}$. We have therefore proved:

THEOREM 10.1. Let $G$ be compact and connected, and let $H$ be connected. Assume that the field is complex. Let $\mathscr{K}_{i}$ be the sheaf of representative elements of type $M_{i}$ in the kernel of an invariant differential operator $\mathscr{D}: \mathscr{V} \rightarrow \mathscr{W}$. Let $K_{i}$ be the submodule of representative elements of type $M_{i}$ in the kernel of the corresponding invariant homomorphism. Then the cohomology of $B$ with coefficients in the sheaf $\mathscr{K}_{i}$ is isomorphic to $H\left(\mathrm{~g}^{*}, \mathfrak{h}^{*}\right) \otimes K_{i}$ if $i \in J$, i.e., if $M_{i}$ is a simple $G$-module, and is zero if $i \notin J$.

Let $K_{R G}=\sum_{J} K_{i}$, so that $K_{R G}$ consists of all representative elements in the kernel of the invariant homomorphism which generate a semisimple $g$-module, this representation of $\mathrm{g}$ being induced by one of $G$. The direct sum decomposition $\mathscr{K}_{R}=\sum_{I} \mathscr{K}_{i}$ induces a corresponding decomposition of the sheaf cohomology groups, so we can state:

COROLlaRY. Under the hypotheses of the theorem, the cohomology of $B$ with coefficients in the sheaf $\mathscr{K}_{R}$ of representative elements in the kernel of $\mathscr{D}$ is isomorphic to $H\left(\mathfrak{g}^{*}, \mathfrak{h}^{*}\right) \otimes K_{R G}$.

\section{REFERENCES}

1. R. Bott, "The index theorem for homogeneous differential operators," in Differential and combinatorial topology, edited by S. Cairns, Princeton Univ. Press, Princeton, N. J., 1965, pp. 167-186.

2. C. Chevalley and S. Eilenberg, Cohomology theory of Lie groups and Lie algebras, Trans. Amer. Math. Soc. 63 (1948), 85-124.

3. S. Helgason, Differential geometry and symmetric spaces, Academic Press, New York, 1962.

4. G. Hochschild, Representation theory of Lie algebras (Multigraphed notes), Univ. of Chicago, Chicago, Ill., 1959.

5. D. Spencer, de Rham theorems and Neumann decompositions associated with linear partial differential equations, Ann. Inst. Fourier (Grenoble) 14 (1964), 1-20.

The University of California, Irvine, California 